ESAIM: COCV 21 (2015) 324-347

DOI: $10.1051 / \mathrm{cocv} / 2014019$
ESAIM: Control, Optimisation and Calculus of Variations

www.esaim-cocv.org

\title{
ACOUSTIC WAVE GUIDES AS INFINITE-DIMENSIONAL DYNAMICAL SYSTEMS
}

\author{
Atte Aalto ${ }^{1}$, Teemu Lukkari $^{2}$ And Jarmo Malinen ${ }^{1}$
}

\begin{abstract}
We prove the unique solvability, passivity/conservativity and some regularity results of two mathematical models for acoustic wave propagation in curved, variable diameter tubular structures of finite length. The first of the models is the generalised Webster's model that includes dissipation and curvature of the 1D waveguide. The second model is the scattering passive, boundary controlled wave equation on $3 \mathrm{D}$ waveguides. The two models are treated in an unified fashion so that the results on the wave equation reduce to the corresponding results of approximating Webster's model at the limit of vanishing waveguide intersection.
\end{abstract}

Mathematics Subject Classification. 35L05, 35L20, 93C20, 47N70.

Received December 20, 2012. Revised February 28, 2014.

Published online October 17, 2014.

\section{INTRODUCTION}

This is the second part of the three part mathematical study on acoustic wave propagation in a narrow, tubular 3D domain $\Omega \subset \mathbb{R}^{3}$. The other parts of the work are $[25,26]$. Our current interest in wave guide dynamics stems from modelling of acoustics of speech production; see, e.g., $[1,3,13]$ and the references therein.

The main purpose of the present paper is to give a rigorous treatment of solvability and energy passivity/conservativity questions of the two models for wave propagations that are discussed in detail in [26]: these are (i) the boundary controlled wave equation on a tubular domain, and (ii) the generalised Webster's horn model that approximates the wave equation in low frequencies. The a posteriori error estimate for the Webster's model is ultimately given in [25], and it is in an essential part based on Theorems 4.1 and 5.1 below.

The secondary purpose of this paper is to introduce the new notion of conservative majoration for passive boundary control systems. The underlying systems theory idea is simple and easy to explain: it is to be expected on engineering and physical grounds that adding energy dissipation to a forward time solvable (i.e., internally well-posed, typically even conservative) system cannot make the system ill-posed, e.g., unsolvable in forward time direction. Thus, it should be enough to treat mathematically only the lossless conservative case that "majorates" all models where dissipation is included as far as we are not reversing the arrow of time. That this intuition holds true for many types of energy dissipation is proved in Theorem 3.1 for boundary dissipation and

\footnotetext{
Keywords and phrases. Wave propagation, tubular domain, wave equation, Webster's horn model, passivity, regularity.

1 Aalto University, Dept. Mathematics and Systems Analysis, P.O. Box 11100, 00076 Aalto, Finland. jarmo.malinen@aalto.fi

2 Department of Mathematics and Statistics, P.O. Box 35 (MaD), 40014 University of Jyväskylä, Finland.
} 


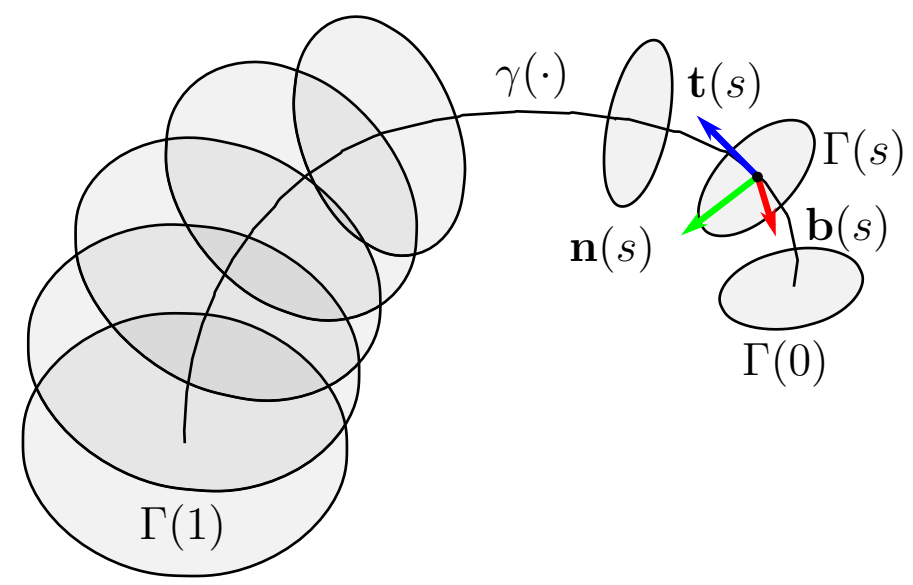

FIGURE 1. The Frenet frame of the planar centreline for a tubular domain $\Omega$, represented by some of its intersection surfaces $\Gamma(s)$ for $s \in[0,1]$. The wall $\Gamma \subset \partial \Omega$ is not shown, and the global coordinate system is detailed in [26], Section 2.

in Theorem 3.2 for a class of dissipation terms for PDE's. These theorems are given in the general context of boundary nodes that have been discussed in, e.g., [29,30,42].

Early work concerning Webster's equation can be found in [5, 40,41,47]. Webster's original work [47] was published in 1919, but the model itself has a longer history spanning over 200 years and starting from the works of Bernoulli, Euler, and Lagrange. More modern approaches are provided by [20,21,31-34]. Webster's horn model is a special case of the wave equation in a non-homogenous medium in $\Omega \subset \mathbb{R}^{n}, n \geq 1$, which has been treated with various boundary and interior point control actions in, e.g. ([9], Appendix 2) ([18], Sect. 2) [22] ([37], Sect. 6) and, in particular ([19], Sect. 7) which contains also historical remarks. There exists a rich literature on the damped wave equation in a 1D spatial domain, and instead of trying to give here a comprehensive account we refer to the numerous references given in [10].

The boundary of $\Omega \subset \mathbb{R}^{n}, n \geq 2$, is smooth or $C^{2}$ in the works cited above, which excludes polygons (for $n=2$ ) or their higher dimensional counterparts such as the tubular structures discussed here. From systems theory point of view, this is a serious restriction since it is obviously impossible to connect finitely many, disjoint, smooth domains seamlessly to each other without leaving holes whose interior is non-empty. The generality of this article makes it possible to interconnect $3 \mathrm{D}$ wave equation systems on geometrically compatible elements $\Omega_{j} \subset \mathbb{R}^{3}$ to form aggregated systems on $\cup_{j} \Omega_{j}$ in the same way as described in ([2], Sect. 5) for Webster's horn model.

Theorems 4.1 and 5.1 treat the questions of unique solvability, passivity, and regularity of the two wave propagation models in the exactly the same form as these results are required in the companion papers $[25,26]$. The strict passivity (i.e., the case $\alpha>0$ ) in Theorems 4.1 and 5.1 could be proved without resorting to Theorems 3.1 and 3.2 as they both concern single PDE's with simple dissipation models. However, the direct approach becomes technically quite cumbersome if we have more complicated aggregated systems to treat (not all of which need be defined by PDE's), and combinations of various dissipation models are involved. An example of such systems is provided by transmission graphs as introduced in [2] where the general passive case is treated by reducing it to the conservative case and arguing as in Theorem 3.2. In the context of transmission graphs, see also the literature on port-Hamiltonian systems $[4,16,46]$ and the abstract boundary spaces [11]. That the conservative majoration method cannot be used for all possible dissipation terms is shown in Section 6 by an example involving Kelvin-Voigt structural damping.

Let us return to wave propagation models on a tubular domain $\Omega$ referring to Figure 1 . The cross sections $\Gamma(s)$ of $\Omega$ are normal to the planar curve $\gamma=\gamma(s)$ that serves as the centreline of $\Omega$ as shown in Figure 1. 
We denote by $R(s)$ and $A(s):=\pi R(s)^{2}$ the radius and the area of $\Gamma(s)$, respectively. We call $\Gamma$ the wall, and the circular plates $\Gamma(0), \Gamma(1)$ the ends of the tube $\Omega$. The boundary of $\Omega$ satisfies $\partial \Omega=\bar{\Gamma} \cup \Gamma(0) \cup \Gamma(1)$. Without loss of generality, the parameter $s \geq 0$ can be regarded as the arc length of $\gamma$, measured from the control/observation surface $\Gamma(0)$ of the tube.

As is well-known, acoustic wave propagation in $\Omega$ can be modelled by the wave equation for the velocity potential $\phi: \Omega \times \overline{\mathbb{R}}^{+} \rightarrow \mathbb{R}$ as

$$
\left\{\begin{array}{l}
\phi_{t t}(\mathbf{r}, t)=c^{2} \Delta \phi(\mathbf{r}, t) \quad \text { for } \mathbf{r} \in \Omega \text { and } t \in \mathbb{R}^{+}, \\
c \frac{\partial \phi}{\partial \nu}(\mathbf{r}, t)+\phi_{t}(\mathbf{r}, t)=2 \sqrt{\frac{c}{\rho A(0)}} u(\mathbf{r}, t) \quad \text { for } \mathbf{r} \in \Gamma(0) \text { and } t \in \mathbb{R}^{+}, \\
\phi(\mathbf{r}, t)=0 \quad \text { for } \mathbf{r} \in \Gamma(1) \text { and } t \in \mathbb{R}^{+}, \\
\alpha \frac{\partial \phi}{\partial t}(\mathbf{r}, t)+\frac{\partial \phi}{\partial \nu}(\mathbf{r}, t)=0 \quad \text { for } \mathbf{r} \in \Gamma, \text { and } t \in \mathbb{R}^{+}, \text {and } \\
\phi(\mathbf{r}, 0)=\phi_{0}(\mathbf{r}), \quad \rho \phi_{t}(\mathbf{r}, 0)=p_{0}(\mathbf{r}) \quad \text { for } \mathbf{r} \in \Omega
\end{array}\right.
$$

with the observation defined by

$$
c \frac{\partial \phi}{\partial \nu}(\mathbf{r}, t)-\phi_{t}(\mathbf{r}, t)=2 \sqrt{\frac{c}{\rho A(0)}} y(\mathbf{r}, t) \quad \text { for } \mathbf{r} \in \Gamma(0) \text { and } t \in \mathbb{R}^{+},
$$

where $\mathbb{R}^{+}=(0, \infty), \overline{\mathbb{R}}^{+}=[0, \infty), \nu$ denotes the unit normal vector on $\partial \Omega, c$ is the sound speed, $\rho$ is the density of the medium, and $\alpha \geq 0$ is a parameter associated to boundary dissipation. The functions $u$ and $y$ are control and observation signals in scattering form, and the normalisation constant $2 \sqrt{\frac{c}{\rho A(0)}}$ takes care of their physical dimension which is power per area. Solvability, stability, and energy questions for the wave equation in various geometrical domains $\Omega \subset \mathbb{R}^{n}$ have a huge literature, and it is not possible to give a historically accurate review here. The wave equation is a prototypal example of a linear hyperbolic PDE whose classical mathematical treatment can be found, e.g., in ([23], Chap. 5) and the underlying physics is explained well in ([8], Chap. 9). In the operator and mathematical system theory context, it has been given as an example (in various variations) in $[27,30,43,44,48]$ and elsewhere. For applications in speech research, see, e.g., $[3,13,26]$ and the references therein.

One computationally and analytically simpler wave propagation model is the generalised Webster's horn model for the same tubular domain $\Omega$ that is now represented by the area function $A(\cdot)$ introduced above. To review this model in its generalised form, let us recall some notions from [26]. To take into account the curvature $\kappa(s)$ of the centreline $\gamma(\cdot)$ of $\Omega$, we adjust the sound speed $c$ in (1.1) by defining $c(s):=c \Sigma(s)$ where $\Sigma(s):=\left(1+\frac{1}{4} \eta(s)^{2}\right)^{-1 / 2}$ is the sound speed correction factor, and $\eta(s):=R(s) \kappa(s)$ is the curvature ratio at $s \in[0,1]$. We also need take into consideration the deformation of the outer wall $\Gamma$ by defining the stretching factor $W(s):=R(s) \sqrt{R^{\prime}(s)^{2}+(\eta(s)-1)^{2}}$ (see [26], Eq. (2.8)). It is a standing assumption that $\eta(s)<1$ to prevent the tube $\Omega$ from folding on itself locally.

Following [26], the generalised Webster's horn model for the velocity potential $\psi:[0,1] \times \overline{\mathbb{R}}^{+} \rightarrow \mathbb{R}$ is now given by

$$
\left\{\begin{array}{l}
\psi_{t t}=\frac{c(s)^{2}}{A(s)} \frac{\partial}{\partial s}\left(A(s) \frac{\partial \psi}{\partial s}\right)-\frac{2 \pi \alpha W(s) c(s)^{2}}{A(s)} \frac{\partial \psi}{\partial t} \\
\text { for } s \in(0,1) \text { and } t \in \mathbb{R}^{+}, \\
-c \psi_{s}(0, t)+\psi_{t}(0, t)=2 \sqrt{\frac{c}{\rho A(0)}} \tilde{u}(t) \quad \text { for } t \in \mathbb{R}^{+}, \\
\psi(1, t)=0 \quad \text { for } t \in \mathbb{R}^{+}, \quad \text { and } \\
\psi(s, 0)=\psi_{0}(s), \quad \rho \psi_{t}(s, 0)=\pi_{0}(s) \quad \text { for } s \in(0,1),
\end{array}\right.
$$


and the observation $\tilde{y}$ is defined by

$$
-c \psi_{s}(0, t)-\psi_{t}(0, t)=2 \sqrt{\frac{c}{\rho A(0)}} \tilde{y}(t) \quad \text { for } t \in \mathbb{R}^{+} .
$$

The constants $c, \rho, \alpha$ are same as in (1.1). The input and output signals $\tilde{u}$ and $\tilde{y}$ of (1.3), (1.4) correspond to $u$ and $y$ in (1.1), (1.2) by spatial averaging over the control surface $\Gamma(0)$. Hence, their physical dimension is power per area as well. Based on $[25,26]$, the solution $\psi$ of $(1.3)$ approximates the averages

$$
\bar{\phi}(s, t):=\frac{1}{A(s)} \int_{\Gamma(s)} \phi \mathrm{d} A \quad \text { for } \quad s \in(0,1) \quad \text { and } \quad t \geq 0
$$

of $\phi$ in (1.1) when $\phi$ is regular enough. Note that the dissipative boundary condition $\alpha \frac{\partial \phi}{\partial \nu}(\mathbf{r}, t)+\frac{\partial \phi}{\partial \nu}(\mathbf{r}, t)=0$ in (1.1) has been replaced by the dissipation term $2 \pi \alpha W(s) A(s)^{-1} c(s)^{2} \frac{\partial \psi}{\partial t}$ (with the same parameter $\alpha$ ) in (1.3). For classical work on Webster's horn model, see [20,31,40] and in particular [33] where numerous references can be found.

We show in Theorem 5.1 that the wave equation model (1.1), (1.2) is uniquely solvable in forward directions of time, and the solution satisfies an energy inequality if $\alpha>0$. By Corollary 5.2, the model has the same properties for $\alpha=0$ but then the energy inequality is replaced by an equality, and the model is even time-flow invertible. In all cases, the solution $\phi$ is observed to have the regularity required for the treatment given in [26] if the input $u$ is twice continuously differentiable. The generalised Webster's horn model (1.3), (1.4) is treated in a similar manner in Theorem 4.1.

This paper is organised as follows: Background on boundary control systems is given in Section 2. Conservative majoration of passive boundary control systems is treated in Section 3. The Webster's horn model and the wave equation are treated in Sections 4 and 5 respectively. Some immediate extensions of these results are given in Section 6. Because of the lack of accessible, complete, and sufficiently general references, the paper is completed by a self-contained appendix on Sobolev spaces, boundary trace operators, Green's identity, and Poincaré inequality for special Lipschitz domains that are required in the rigorous analysis of typical wave guide geometries.

\section{ON INFINITE DIMENSIONAL SYSTEMS}

Linear boundary control systems such as (1.1) and (1.3) are treated as dynamical systems that can be described by operator differential equations of the form

$$
u(t)=G z(t), \quad \dot{z}(t)=L z(t), \quad \text { with the initial condition } \quad z(0)=z_{0}
$$

and the observation equation

$$
y(t)=K z(t),
$$

where $t \in \overline{\mathbb{R}}^{+}=[0, \infty)$ denotes time. The signals in (2.1), (2.2) are as follows: $u$ is the input, $y$ is the output, and the state trajectory is $z$.

\section{Cauchy problems}

To make (2.1) properly solvable for all twice differentiable $u$ and compatible initial states $z_{0}$, the axioms of an internally well-posed boundary node should be satisfied:

Definition 2.1. A triple of operators $\Xi=(G, L, K)$ is an internally well-posed boundary node on the Hilbert spaces $(\mathcal{U}, \mathcal{X}, \mathcal{Y})$ if the following conditions are satisfied:

(i) $G, L$, and $K$ are linear operators with the same domain $\mathcal{Z} \subset \mathcal{X}$; 
(ii) $\left[\begin{array}{l}G \\ L \\ K\end{array}\right]$ is a closed operator from $\mathcal{X}$ into $\mathcal{U} \times \mathcal{X} \times \mathcal{Y}$ with domain $\mathcal{Z}$;

(iii) $G$ is surjective, and $\operatorname{ker}(G)$ is dense in $\mathcal{X}$; and

(iv) $\left.L\right|_{\operatorname{ker}(G)}$ (understood as an unbounded operator in $\mathcal{X}$ with domain $\operatorname{ker}(G)$ ) generates a strongly continuous semigroup on $\mathcal{X}$.

If, in addition, $L$ is a closed operator on $\mathcal{X}$ with domain $\mathcal{Z}$, we say that the boundary node $\Xi$ is strong.

The history of abstract boundary control systems dates back to $[7,38,39]$. The phrase "internally well-posed" refers to condition (iv) of Definition 2.1, and it is a much weaker property than well-posedness of systems in the sense of [42]. It plainly means that the boundary node defines an evolution equation that is uniquely solvable in forward time direction. Boundary nodes that are not necessarily internally well-posed are characterised by the weaker requirement in place of (iv): $\alpha-\left.L\right|_{\operatorname{ker}(G)}$ is a bijection from $\operatorname{ker}(G)$ onto $\mathcal{X}$ for some $\alpha \in \mathbb{C}$.

We call $\mathcal{U}$ the input space, $\mathcal{X}$ the state space, $\mathcal{Y}$ the output space, $\mathcal{Z}$ the solution space, $G$ the input boundary operator, $L$ the interior operator, and $K$ the output boundary operator. The operator $A:=\left.L\right|_{\operatorname{ker}(G)}$ is called the semigroup generator if $\Xi$ is internally well-posed, and otherwise it is known as the main operator of $\Xi$. Because $\left[\begin{array}{lll}G & L & K\end{array}\right]^{T}$ is a closed operator, we can give its domain the Hilbert space structure by the graph norm

$$
\|z\|_{\mathcal{Z}}^{2}=\|z\|_{\mathcal{X}}^{2}+\|L z\|_{\mathcal{X}}^{2}+\|G z\|_{\mathcal{U}}^{2}+\|K z\|_{\mathcal{Y}}^{2} .
$$

If the node is strong, we have an equivalent norm for $\mathcal{Z}$ given by omitting the last two terms in (2.3). If $\Xi=(G, L, K)$ is an internally well-posed boundary node, then (2.1) has a unique "smooth" solution:

Proposition 2.2. Assume that $\Xi=(G, L, K)$ is an internally well-posed boundary node. For all $z_{0} \in \mathcal{X}$ and $u \in C^{2}\left(\overline{\mathbb{R}}^{+} ; \mathcal{U}\right)$ with $G z_{0}=u(0)$ the equations $(2.1)$ have a unique solution $z \in C^{1}\left(\overline{\mathbb{R}}^{+} ; \mathcal{X}\right) \cap C\left(\overline{\mathbb{R}}^{+} ; \mathcal{Z}\right)$. Hence, the output $y \in C\left(\overline{\mathbb{R}}^{+} ; \mathcal{Y}\right)$ is well defined by the equation $(2.2)$.

Indeed, this is [29], Lemma 2.6 .

\section{Energy balances}

Now that we have treated the solvability of the dynamical equations, it remains to consider energy notions. We say that the internally well-posed boundary node $\Xi=(G, L, K)$ is (scattering) passive if all smooth solutions of (2.1) satisfy

$$
\frac{\mathrm{d}}{\mathrm{d} t}\|z(t)\|_{\mathcal{X}}^{2}+\|y(t)\|_{\mathcal{Y}}^{2} \leq\|u(t)\|_{\mathcal{U}}^{2} \quad \text { for all } \quad t \in \mathbb{R}^{+}
$$

with $y$ given by (2.2). All such systems are well-posed in the sense of [42]; see also [45]. We say that $\Xi$ is (scattering) energy preserving if (2.4) holds as an equality.

Many boundary nodes arising from hyperbolic PDE's (such as (1.1), (1.2) and (1.3), (1.4) for $\alpha=0)$ have the property that they remain boundary nodes if we (i) change the sign of $L$ (i.e., reverse the direction of time); and (ii) interchange the roles of $K$ and $G$ (i.e., reverse the flow direction). Such boundary nodes are called time-flow invertible, and we write $\Xi^{\leftarrow}=(K,-L, G)$ for the time-flow inverse of $\Xi$. There are many equivalent definitions of conservativity in the literature, and we choose here the following:

Definition 2.3. An internally well-posed boundary node $\Xi$ is (scattering) conservative if it is time-flow invertible, and both $\Xi$ itself and the time-flow inverse $\Xi^{\leftarrow}$ are (scattering) energy preserving ${ }^{3}$.

For system nodes that have been introduced in $[28,42]$, an equivalent definition for conservativity is to require that both $S$ and its dual node $S^{d}$ are energy preserving. This is the straightforward generalisation from the finite-dimensional theory but it is not very practical when dealing with boundary control. For conservative systems, the time-flow inverse and the dual system coincide, and we have then, in particular, $A^{*}=-\left.L\right|_{\operatorname{ker}(K)}$ if $A=\left.L\right|_{\operatorname{ker}(G)}$. For details, see [29], Theorems 1.7 and 1.9] It is possible to check economically, without directly

\footnotetext{
${ }^{3}$ The words "energy preserving" can be replaced by "passive" without changing the class of systems one obtains.
} 
using Definition 2.1, that the triple $\Xi=(G, L, K)$ is a dissipative/conservative boundary node:

Proposition 2.4. Let $\Xi=(G, L, K)$ be a triple of linear operators with a common domain $\mathcal{Z} \subset \mathcal{X}$, and ranges in the Hilbert spaces $\mathcal{U}, \mathcal{X}$, and $\mathcal{Y}$, respectively. Then $\Xi$ is a passive boundary node on $(\mathcal{U}, \mathcal{X}, \mathcal{Y})$ if and only if the following conditions hold:

(i) We have the Green-Lagrange inequality

$$
2 \operatorname{Re}\langle z, L z\rangle_{\mathcal{X}}+\|K z\|_{\mathcal{Y}}^{2} \leq\|G z\|_{\mathcal{U}}^{2} \quad \text { for all } \quad z \in \mathcal{Z}
$$

(ii) $G \mathcal{Z}=\mathcal{U}$ and $(\beta-L) \operatorname{ker}(G)=\mathcal{X}$ for some $\beta \in \mathbb{C}^{+}$(hence, for all $\beta \in \mathbb{C}^{+}$).

Similarly, $\Xi$ is a conservative boundary node on $(\mathcal{U}, \mathcal{X}, \mathcal{Y})$ if and only if (ii) above holds together with the additional conditions:

(iii) We have the Green-Lagrange identity

$$
2 \operatorname{Re}\langle z, L z\rangle_{\mathcal{X}}+\|K z\|_{\mathcal{Y}}^{2}=\|G z\|_{\mathcal{U}}^{2} \quad \text { for all } \quad z \in \mathcal{Z} .
$$

(iv) $K \mathcal{Z}=\mathcal{Y}$ and $(\gamma+L) \operatorname{ker}(K)=\mathcal{X}$ for some $\gamma \in \mathbb{C}^{+}$(hence, for all $\left.\gamma \in \mathbb{C}^{+}\right)$.

This is a slight modification of ([30], Thm. 2.5); see also ([29], Prop. 2.5).

\section{Conservative majorants}

In some applications, energy dissipation in a linear dynamical system is often caused by a distinct part of the model such as a term or a boundary condition imposed on the defining PDE. If this part is completely removed from the model, the resulting more simple system is conservative and, in particular, internally well-posed. We call it a conservative majorant of the original system.

Intuition from engineering and physics hints that increasing dissipation should make the system "better behaved" and not spoil the internal well-posedness ${ }^{4}$. The following Theorems 3.1 and 3.2 apply to many boundary control systems. However, they are written for passive majorants since the proofs remain the same, and this way the results can be applied successively to systems having both boundary dissipation and dissipative terms.

Theorem 3.1. Let $\underset{\widetilde{\Xi}}{\widetilde{\mathcal{G}}}=\left(\left[\begin{array}{c}G \\ \tilde{G}\end{array}\right], L,\left[\begin{array}{c}K \\ \tilde{K}\end{array}\right]\right)$ be a scattering passive boundary node on Hilbert spaces $(\mathcal{U} \oplus \tilde{\mathcal{U}}, \mathcal{X}, \mathcal{Y} \oplus \tilde{\mathcal{Y}})$ with solution space $\tilde{\mathcal{Z}}$. Then $\Xi:=\left(\left.G\right|_{\mathcal{Z}},\left.L\right|_{\mathcal{Z}},\left.K\right|_{\mathcal{Z}}\right)$ is a scattering passive boundary node on $(\mathcal{U}, \mathcal{X}, \mathcal{Y})$ with the solution space $\mathcal{Z}:=\operatorname{ker}(\tilde{G})$. Both $\widetilde{\Xi}$ and $\Xi$ have the same semigroup generator, namely $\left.L\right|_{\operatorname{ker}(G) \cap \operatorname{ker}(\tilde{G})}$. If $\widetilde{\Xi}$ is a strong node, so is $\Xi$.

Proof. The Green-Lagrange inequality holds for $\Xi$ since for $z \in \operatorname{ker}(\tilde{G})$ we have $\|G z\|_{\mathcal{U}}=\left\|\left[\begin{array}{c}G \\ \tilde{G}\end{array}\right] z\right\|_{\mathcal{U} \oplus \tilde{\mathcal{U}}}$, and hence we get by the passivity of $\widetilde{\Xi}$

$$
2 \operatorname{Re}\langle z, L z\rangle_{\mathcal{X}}-\|G z\|_{\mathcal{U}}^{2} \leq-\left\|\left[\begin{array}{c}
K z \\
\tilde{K} z
\end{array}\right]\right\|_{\mathcal{Y} \oplus \tilde{\mathcal{Y}}}^{2} \leq-\|K z\|_{\mathcal{Y}}^{2} .
$$

The surjectivity $G \mathcal{Z}=\mathcal{U}$ follows from $\mathcal{U} \oplus\{0\} \subset \mathcal{U} \oplus \tilde{\mathcal{U}}=\left[\begin{array}{c}G \\ \tilde{G}\end{array}\right] \mathcal{Z}$ and $\mathcal{Z}=\operatorname{ker}(\tilde{G})$. Since $(\beta-L) \operatorname{ker}\left(\left.G\right|_{\mathcal{Z}}\right)=$ $\left.(\beta-L)\right|_{\operatorname{ker}(\tilde{G})} \operatorname{ker}(G)=(\beta-L)(\operatorname{ker}(G) \cap \operatorname{ker}(\tilde{G}))=(\beta-L) \operatorname{ker}\left(\left[\begin{array}{c}G \\ \tilde{G}\end{array}\right]\right)=\mathcal{X}$, the passivity of $\Xi$ follows by Proposition 2.4 .

Suppose that $L$ is closed (i.e., $\widetilde{\Xi}$ is strong) and that $\tilde{\mathcal{Z}} \supset \mathcal{Z} \ni z_{j} \rightarrow z$ in $\mathcal{X}$ is such that $L z_{j} \rightarrow x$ in $\mathcal{X}$ as $j \rightarrow \infty$. Because $L$ is closed, $z \in \operatorname{dom}(L)=\tilde{\mathcal{Z}}$ and $L z=x$. Thus, $\left\|z_{j}-z\right\|_{\mathcal{Z}}^{2}:=\left\|z_{j}-z\right\|_{\mathcal{X}}^{2}+\left\|L\left(z_{j}-z\right)\right\|_{\mathcal{X}}^{2} \rightarrow 0$. Because $\tilde{G} \in \mathcal{L}(\mathcal{Z} ; \tilde{\mathcal{U}})$ by applying $(2.3)$ on $\widetilde{\Xi}$, the space $\mathcal{Z}=\operatorname{ker}(\tilde{G})$ is closed in $\tilde{\mathcal{Z}}$ and thus $z \in \mathcal{Z}$. We have now shown that $\left.L\right|_{\mathcal{Z}}$ is closed with $\operatorname{dom}\left(\left.L\right|_{\mathcal{Z}}\right)=\mathcal{Z}$.

\footnotetext{
${ }^{4}$ The dissipativity or even the internal well-posedness of the time-flow inverted system is, if course, destroyed since adding dissipation creates the "arrow of time".
} 
The restriction of the original solution space to $\operatorname{ker}(\tilde{G})$ in Theorem 3.1 is a functional analytic description of boundary dissipation of a particular kind. If the original scattering passive $\widetilde{\Xi}$ is translated to an impedance passive boundary node by the external Cayley-transform (see [30], Def. 3.1), then the abstract boundary condition by restriction to $\operatorname{ker}(\tilde{G})$ can be understood as a termination to an ideally resistive element as depicted in Figure 1 of [30].

Theorem 3.2. Let $\Xi=(G, L, K)$ be a scattering passive boundary node on the Hilbert spaces $(\mathcal{U}, \mathcal{X}, \mathcal{Y})$ with solution space $\mathcal{Z}$ and $\mathcal{X}_{1}=\operatorname{ker}(G)$ with the norm $\|z\|_{\mathcal{X}_{1}}=\|(1-L) z\|_{\mathcal{X}}$. Let $H$ be a dissipative operator on $\mathcal{X}$ with $\mathcal{Z} \subset \operatorname{dom}(H)^{5}$. Introduce two assumptions as follows:

(i) There is $a>0$ and $0 \leq b<1$ such that $\|H z\|_{\mathcal{X}} \leq a\|z\|_{\mathcal{X}}+b\|L z\|_{\mathcal{X}}$ for all $z \in \operatorname{ker}(G)$.

(ii) There is a Hilbert space $\tilde{\mathcal{X}}$ such that $\mathcal{X}_{1} \subset \tilde{\mathcal{X}} \subset \operatorname{dom}(H)$, the inclusion $\mathcal{X}_{1} \subset \tilde{\mathcal{X}}$ is compact and $\left.H\right|_{\tilde{\mathcal{X}}} \in$ $\mathcal{L}(\tilde{\mathcal{X}} ; \mathcal{X})$.

If either (i) or (ii) holds, then $\Xi_{H}:=(G, L+H, K)$ is a scattering passive boundary node. We have dom $(A)=$ $\operatorname{dom}\left(A_{H}\right)$ where $A=\left.L\right|_{\operatorname{ker}(G)}$ and $A_{H}=\left.(L+H)\right|_{\operatorname{ker}(G)}$ are the semigroup generators of $\Xi$ and $\Xi_{H}$, respectively. If the node $\Xi$ is strong and $H \in \mathcal{L}(\mathcal{X})$ (i.e., $b=0$ in assumption (i)), then $\Xi_{H}$ is a strong boundary node as well.

Both the assumptions (i) and (ii) hold if $H \in \mathcal{L}(\mathcal{X})$ and $\mathcal{X}_{1} \subset \mathcal{X}$ with a compact inclusion. This is the case in ([2], Sect. 5) in the context of an impedance passive system. The compactness property is typically a consequence of the Rellich-Kondrachov Theorem ([6], Thm. 1, p. 144) for boundary nodes defined by PDE's on bounded domains. In many applications such as Theorem 4.1 below, the operator $H$ is even self-adjoint. We give an example of the 1D wave equation with Kelvin-Voigt damping in Section 6 where Theorem 3.2 cannot be applied.

Proof. By using assumption (i): This argument is motivated by ([14], Thm. 2.7, p. 501). Let us first show that $A_{H}:=A+\left.H\right|_{\operatorname{ker}(G)}$ with $\operatorname{dom}\left(A_{H}\right)=\operatorname{ker}(G)$ generates a contraction semigroup on $\mathcal{X}$ where $A=\left.L\right|_{\operatorname{ker}(G)}$ generates the contraction semigroup of $\Xi$ as usual. As a first step, we establish the inequality $\left\|H(s-A)^{-1}\right\|_{\mathcal{L}(\mathcal{X})}<1$ for all real $s$ large enough.

Let $\beta>0$ be arbitrary. For all $s>\beta$ and $z \in \mathcal{X}$ we have

$$
\begin{aligned}
\left\|H(s-A)^{-1} z\right\|_{\mathcal{X}} \leq & a\left\|(s-A)^{-1} z\right\|_{\mathcal{X}}+b\left\|A(s-A)^{-1} z\right\|_{\mathcal{X}} \\
\leq & (a+\beta b)\left\|(s-A)^{-1} z\right\|_{\mathcal{X}} \\
& +\frac{b}{s-\beta}\left\|\left(\frac{1}{s-\beta}-(A-\beta)^{-1}\right)^{-1} z\right\|_{\mathcal{X}}
\end{aligned}
$$

since

$$
-A(s-A)^{-1}=\frac{1}{s-\beta}\left(\frac{1}{s-\beta}-(A-\beta)^{-1}\right)^{-1}-\beta(s-A)^{-1} .
$$

Since $A$ is a maximally dissipative operator on $\mathcal{X}$, we have for all $z=(A-\beta) x \in \mathcal{X}$ with $x \in \operatorname{dom}(A)$

$$
\begin{aligned}
\operatorname{Re}\left\langle(A-\beta)^{-1} z, z\right\rangle_{\mathcal{X}} & =\operatorname{Re}\left\langle(A-\beta)^{-1}(A-\beta) x,(A-\beta) x\right\rangle_{\mathcal{X}} \\
& =\operatorname{Re}\langle x,(A-\beta) x\rangle_{\mathcal{X}} \\
& =\operatorname{Re}\langle x, A x\rangle_{\mathcal{X}}-\beta\|x\|_{\mathcal{X}}^{2} \leq 0 .
\end{aligned}
$$

Thus, the operator $(A-\beta)^{-1}$ is dissipative, and it is maximally so because $(A-\beta)^{-1} \in \mathcal{L}(\mathcal{X})$.

\footnotetext{
${ }^{5}$ This means that $H: \operatorname{dom}(H) \subset \mathcal{X} \rightarrow \mathcal{X}$ is an operator satisfying $\mathcal{Z} \subset \operatorname{dom}(H)$ and $\operatorname{Re}\langle z, H z\rangle_{\mathcal{X}} \leq 0$ for all $z \in \mathcal{Z}$.
} 
Because $(A-\beta)^{-1}$ generates a $C_{0}$ contraction semigroup on $X$, the Hille-Yoshida generator theorem gives the resolvent estimate

$$
\frac{1}{s-\beta}\left\|\left(\frac{1}{s-\beta}-(A-\beta)^{-1}\right)^{-1}\right\|_{\mathcal{L}(\mathcal{X})} \leq 1
$$

for $s>\beta>0$. Similarly, $\left\|(s-A)^{-1}\right\|_{\mathcal{L}(\mathcal{X})} \leq 1 / s$ for $s>0$. These together with (3.1) give

$$
\frac{\left\|H(s-A)^{-1} z\right\|_{\mathcal{X}}}{\|z\|_{\mathcal{X}}} \leq \frac{a+\beta b}{s}+b<1 \text { for all } s>\frac{a+\beta b}{1-b} .
$$

Because $\beta>0$ was arbitrary, we get $\left\|H(s-A)^{-1}\right\|_{\mathcal{L}(\mathcal{X})}<1$ for all $s>\frac{a}{1-b}$. We conclude that $(a /(1-b), \infty) \subset$ $\rho\left(A_{H}\right)$ and

$$
\left(s-A_{H}\right)^{-1}=(s-A)^{-1}\left(I-H(s-A)^{-1}\right)^{-1}
$$

where $\operatorname{dom}\left(A_{H}\right)=\operatorname{dom}(A)=\operatorname{ker}(G)$. In particular, we have shown that $(2 a /(1-b)-L-H) \operatorname{ker}(G)=\mathcal{X}$ (that $G \mathcal{Z}=\mathcal{U}$ holds, follows because $\Xi$ itself is a boundary node with the same input boundary operator $G$ ). Since the Green-Lagrange inequality (2.5) holds by the passivity of $\Xi$ and $\operatorname{Re}\langle z, H z\rangle_{\mathcal{X}} \leq 0$ by assumption, we conclude that (2.5) holds with $L+H$ in place of $L$, too. Thus $\Xi_{H}$ is a scattering passive boundary node by Proposition 2.4.

By using assumption (ii): As in the first part of this proof, it is enough to prove that $\rho\left(A_{H}\right) \cap \mathbb{C}_{+} \neq \emptyset$ by verifying (3.2). Because $(s-A)^{-1} \in \mathcal{L}\left(\mathcal{X} ; \mathcal{X}_{1}\right), \mathcal{X}_{1} \subset \tilde{\mathcal{X}}$ is compact, and $\left.H\right|_{\tilde{\mathcal{X}}} \in \mathcal{L}(\tilde{\mathcal{X}} ; \mathcal{X})$, we conclude that $H(s-A)^{-1} \in \mathcal{L}(\mathcal{X})$ is a compact operator for all $s \in \mathbb{C}_{+}$. If there is a $s>0$ such that $1 \notin \sigma\left(H(s-A)^{-1}\right) \subset$ $\sigma_{p}\left(H(s-A)^{-1}\right) \cup\{0\}$, then (3.2) holds, $s \in \rho\left(A_{H}\right)$, and $\Xi_{H}$ is a passive boundary node as argued in the first part of the proof. For contradiction, assume that $1 \in \sigma_{p}\left(H\left(s_{0}-A\right)^{-1}\right)$ for some $s_{0}>0$. This implies $A_{H} x_{0}=s_{0} x_{0}$ for some $x_{0} \in \operatorname{dom}\left(A_{H}\right)$, and hence

$$
\operatorname{Re}\left\langle A_{H} x_{0}, x_{0}\right\rangle_{\mathcal{X}}=s_{0}\left\|x_{0}\right\|_{\mathcal{X}}^{2}>0
$$

which contradicts the dissipativity of $A_{H}=A+\left.H\right|_{\operatorname{ker}(G)}$. Thus (3.2) holds and $\operatorname{dom}(A)=\operatorname{dom}\left(A_{H}\right)$. The final claim about strongness of $\Xi_{H}$ holds because perturbations of closed operators by bounded operators are closed.

The perturbation $H$ in Theorem 3.2 is a densely defined dissipative operator on $\mathcal{X}$. As such, it has a maximally dissipative (closed) extension $\widetilde{H}: \operatorname{dom}(\widetilde{H}) \subset \mathcal{X} \rightarrow \mathcal{X}$ satisfying $\widetilde{H}^{*} \subset H^{*}$, and the adjoint $\widetilde{H}^{*}$ is maximally dissipative as well. Without loss of generality we may assume that $H=\widetilde{H}$ in Theorem 3.2. Furthermore, it is possible to use $\tilde{\mathcal{X}}=\operatorname{dom}(\widetilde{H})$ equipped with the graph norm $\|z\|_{\operatorname{dom}(\widetilde{H})}^{2}=\|z\|_{\mathcal{X}}^{2}+\|\widetilde{H} z\|_{\mathcal{X}}^{2}$ in assumption (ii), and it only remains to check whether $\mathcal{X}_{1} \subset \operatorname{dom}(\widetilde{H})$ compactly.

Let us consider the adjoint semigroup of the passive boundary node $\Xi_{H}=(G, L+H, K)$, majorated by the conservative node $\Xi=(G, L, K)$. The adjoint semigroup is generated by the maximally dissipative operator $A_{H}^{*}$ where $A_{H}=\left.(L+H)\right|_{\operatorname{ker}(G)}$ is maximally dissipative under the assumptions of Theorem 3.2.

Proposition 3.3. Let $\Xi=(G, L, K)$ be a scattering conservative boundary node on Hilbert spaces $(\mathcal{U}, \mathcal{X}, \mathcal{Y})$ with solution space $\mathcal{Z}$. Let $H$ be a dissipative operator on $\mathcal{X}$ with $\mathcal{Z} \subset \operatorname{dom}(H)$. Assume that either of the assumptions (i) or (ii) of Theorem 3.2 holds, and let the extension $\widetilde{H}$ be defined as above.

(i) If $\operatorname{ker}(K) \subset \operatorname{dom}\left(\widetilde{H}^{*}\right)$, then $\left.\left(-L+\widetilde{H}^{*}\right)\right|_{\operatorname{ker}(K)} \subset A_{H}^{*}$.

(ii) If $\mathcal{Z} \subset \operatorname{dom}\left(\widetilde{H}^{*}\right)$, then $\Xi_{\widetilde{H}^{*}}^{\overleftarrow{ }}:=\left(K,-L+\widetilde{H}^{*}, G\right)$ is an internally well-posed boundary node if and only if $\left.\left(-L+\widetilde{H}^{*}\right)\right|_{\operatorname{ker}(K)}=A_{H}^{*}$.

(iii) If $\mathcal{Z} \subset \operatorname{dom}\left(\widetilde{H}^{*}\right)$, then $\Xi_{\widetilde{H}^{*}}$ is a passive boundary node if and only if $\left.\left(-L+\widetilde{H}^{*}\right)\right|_{\operatorname{ker}(K)}=A_{H}^{*}$. 
If $\Xi=(G, L, K)$ is conservative, so is its time-flow inverse $\Xi^{\leftarrow}=(K,-L, G)$ by Definition 2.3. In this case, it may be possible to use Theorem 3.2 to conclude that $\Xi_{\overleftarrow{H}^{*}}$ is a passive boundary node as well. If both $\Xi_{H}$ and $\Xi_{\widetilde{H}^{*}}^{\overleftarrow{ }}$ are passive, then they cannot be time-flow inverses of each other unless both nodes are, in fact, conservative; i.e., $H=\widetilde{H}^{*}=0$ on $\mathcal{Z}$.

Proof. It is easy to see that $A^{*}+T^{*} \subset(A+T)^{*}$ holds for operators $A, T$ on $\mathcal{X}$ with $\operatorname{dom}(A) \cap \operatorname{dom}(T)$ dense in $\mathcal{X}$. Applying this on $A=\left.L\right|_{\operatorname{ker}(G)}$ and $T:=\left.\widetilde{H}\right|_{\operatorname{ker}(G)}$ we get on $\operatorname{ker}(K)$ the inclusion $-\left.L\right|_{\operatorname{ker}(K)}+\left(\left.\widetilde{H}\right|_{\operatorname{ker}(G)}\right)^{*} \subset A_{H}^{*}$. Here we used $A^{*}=-\left.L\right|_{\operatorname{ker}(K)}$ which holds because $\Xi=(G, L, K)$ is a conservative boundary node whose dual system (with semigroup generator $A^{*}$ ) coincides with the time-flow inverse $\Xi^{\leftarrow}=(K,-L, G)$. Since $\operatorname{ker}(K) \subset \operatorname{dom}\left(\widetilde{H}^{*}\right)$ has been assumed, it follows that $\left(\left.\widetilde{H}\right|_{\operatorname{ker}(G)}\right)^{*} z=\widetilde{H}^{*} z$ for all $z \in \operatorname{ker}(K)$, and Claim (i) now follows.

The "only if" part of Claims (ii) and (iii): By the internal well-posedness of $\Xi_{\widetilde{H}^{*}}^{\overleftarrow{ }}$, its main operator $(-L+$ $\left.\widetilde{H}^{*}\right)\left.\right|_{\operatorname{ker}(K)}$ generates a $C_{0}$ semigroup, and its resolvent set contains some right half plane by the Hille-Yoshida theorem. By Claim (i) and the fact that $A_{H}^{*}$ is (even maximally) dissipative, it follows that $\left.\left(-L+\widetilde{H}^{*}\right)\right|_{\operatorname{ker}(K)}$ is dissipative. But then $\left.\left(-L+\widetilde{H}^{*}\right)\right|_{\operatorname{ker}(K)}$ is maximally dissipative, and the converse inclusion $A_{H}^{*} \subset(-L+$ $\left.\widetilde{H}^{*}\right)\left.\right|_{\operatorname{ker}(K)}$ follows.

The "if" part of Claim (ii): The operator $\left.\left(-L+\widetilde{H}^{*}\right)\right|_{\operatorname{ker}(K)}$ generates a contraction semigroup on $\mathcal{X}$ because it equals by assumption $A_{H}^{*}$ where $A_{H}$ itself is a generator of a contraction semigroup by Theorem 3.2.

Equip the Hilbert space $\operatorname{dom}\left(\widetilde{H}^{*}\right)$ with the graph norm of the closed operator $\widetilde{H}^{*}$. Since $\mathcal{Z} \subset \operatorname{dom}\left(\widetilde{H}^{*}\right)$ has been assumed, and both $\mathcal{Z}$ and $\operatorname{dom}\left(\widetilde{H}^{*}\right)$ are continuously embedded in $\mathcal{X}$, the inclusion $\mathcal{Z} \subset \operatorname{dom}\left(\widetilde{H}^{*}\right)$ is continuous, too. Now $\left.\widetilde{H}^{*}\right|_{\mathcal{Z}} \in \mathcal{L}(\mathcal{Z} ; \mathcal{X})$ follows from $\widetilde{H}^{*} \in \mathcal{L}\left(\operatorname{dom}\left(\widetilde{H}^{*}\right) ; \mathcal{X}\right)$. Since now $-L+\widetilde{H}^{*} \in \mathcal{L}(\mathcal{Z} ; \mathcal{X})$, it follows that $\Xi_{\widetilde{H}^{*}}^{\leftarrow}$ is an internally well-posed boundary node by [29], Proposition 2.5. (You could also argue by verifying Definition 2.1(ii) directly.)

The "if" part of Claim (iii): The "if" part of Claim (ii) gives the internal well-posedness of $\Xi_{\widetilde{H}^{*}}$. To show passivity, only the Green-Lagrange inequality $2 \operatorname{Re}\left\langle z,\left(-L+\widetilde{H}^{*}\right) z\right\rangle_{\mathcal{X}} \leq\|K z\|_{\mathcal{Y}}^{2}-\|G z\|_{\mathcal{U}}^{2}$ is needed. This follows from (2.6) (by the conservativity of $\Xi^{\leftarrow}$ ) and the dissipativity of $\widetilde{H}^{*}$ with $\mathcal{Z} \subset \operatorname{dom}\left(\widetilde{H}^{*}\right)$ (since $\widetilde{H}$ is maximally dissipative).

\section{Generalised Webster's model for Wave Guides}

As proved in [26], we arrive (under some mild technical assumptions on $\Omega$ as explained in [26], Sect. 3) to the following equations for the approximate spatial averages of solutions of (5.1):

$$
\left\{\begin{array}{l}
\psi_{t t}=\frac{c(s)^{2}}{A(s)} \frac{\partial}{\partial s}\left(A(s) \frac{\partial \psi}{\partial s}\right)-\frac{2 \pi \alpha W(s) c(s)^{2}}{A(s)} \frac{\partial \psi}{\partial t} \\
\text { for } s \in(0,1) \text { and } t \in \mathbb{R}^{+}, \\
-c(0) \psi_{s}(0, t)+\psi_{t}(0, t)=2 \sqrt{\frac{c(0)}{\rho A(0)}} \tilde{u}(t) \quad \text { for } t \in \mathbb{R}^{+}, \\
\psi(1, t)=0 \quad \text { for } t \in \mathbb{R}^{+}, \quad \text { and } \\
\psi(s, 0)=\psi_{0}(s), \quad \rho \psi_{t}(s, 0)=\pi_{0}(s) \quad \text { for } s \in(0,1),
\end{array}\right.
$$


and the observation equation averages to

$$
-c(0) \psi_{s}(0, t)-\psi_{t}(0, t)=2 \sqrt{\frac{c(0)}{\rho A(0)}} \tilde{y}(t) \quad \text { for } t \in \mathbb{R}^{+} .
$$

The notation has been introduced in Section 1. Analogously with the wave equation, the solution $\psi$ is called Webster's velocity potential. In ([25], Sect. 3) we add a load function $f(s, t)$ to obtain the PDE $\psi_{t t}=\frac{c(s)^{2}}{A(s)} \frac{\partial}{\partial s}\left(A(s) \frac{\partial \psi}{\partial s}\right)-\frac{2 \pi \alpha W(s) c(s)^{2}}{A(s)} \frac{\partial \psi}{\partial t}+f(s, t)$ because the argument there is based on the feed-forward connection detailed in ([26], Fig. 1). Only the boundary control input is considered here, and it can be treated using boundary nodes.

We assume that the sound speed correction factor $\Sigma(s)$ and the area function $A(s)$ are infinitely differentiable for $s \in[0,1]$, and that the estimates

$$
0<\min _{s \in[0,1]} A(s) \leq \max _{s \in[0,1]} A(s)<\infty \text { and } 0<\min _{s \in[0,1]} c(s) \leq \max _{s \in[0,1]} c(s)<\infty
$$

hold. These are natural assumptions recalling the geometry of the tubular domain $\Omega$. Define the operators

$$
W:=\frac{1}{A(s)} \frac{\partial}{\partial s}\left(A(s) \frac{\partial}{\partial s}\right) \text { and } D:=-\frac{2 \pi W(s)}{A(s)} .
$$

The operator $D$ should be understood as a multiplication operator on $L^{2}(0,1)$ by the strictly negative function $-2 \pi W(\cdot) A(\cdot)^{-1}$. Then the first of the equations in (4.1) can be cast into first order form by using the rule

$$
\psi_{t t}=c(s)^{2}\left(W \psi+\alpha D \psi_{t}\right) \quad \hat{=} \frac{\mathrm{d}}{\mathrm{d} t}\left[\begin{array}{l}
\psi \\
\pi
\end{array}\right]=\left[\begin{array}{cc}
0 & \rho^{-1} \\
\rho c(s)^{2} W & \alpha c(s)^{2} D
\end{array}\right]\left[\begin{array}{l}
\psi \\
\pi
\end{array}\right] .
$$

Henceforth, let

$$
L_{W}:=\left[\begin{array}{cc}
0 & \rho^{-1} \\
\rho c(s)^{2} W & 0
\end{array}\right]: \mathcal{Z}_{W} \rightarrow \mathcal{X}_{W} \text { and } H_{W}:=\left[\begin{array}{cc}
0 & 0 \\
0 & c(s)^{2} D
\end{array}\right]: \mathcal{X}_{W} \rightarrow \mathcal{X}_{W}
$$

where the Hilbert spaces are given by

$$
\begin{aligned}
& \mathcal{Z}_{W}:=\left(H_{\{1\}}^{1}(0,1) \cap H^{2}(0,1)\right) \times H_{\{1\}}^{1}(0,1), \quad \mathcal{X}_{W}:=H_{\{1\}}^{1}(0,1) \times L^{2}(0,1) \\
& \text { where } \quad H_{\{1\}}^{1}(0,1):=\left\{f \in H^{1}(0,1): f(1)=0\right\} .
\end{aligned}
$$

Clearly we have $H_{W} \in \mathcal{L}\left(\mathcal{X}_{W}\right), H_{W}^{*}=H_{W}$, and this operator is negative in the sense that $\left\langle H_{W}\left[\begin{array}{l}z_{1} \\ z_{2}\end{array}\right],\left[\begin{array}{c}z_{1} \\ z_{2}\end{array}\right]\right\rangle_{\mathcal{X}_{W}}=$ $-2 \pi \int_{0}^{1}\left|z_{2}(s)\right|^{2} W(s) c(s)^{2} A(s)^{-1} \mathrm{~d} s \leq 0$. So, the operator $\alpha H_{W}$ for $\alpha>0$ satisfies assumption (i) of Theorem (i) with $b=0$ and also assumption (ii) of the same theorem with $\tilde{\mathcal{X}}=\mathcal{X}$.

The Hilbert spaces $\mathcal{Z}_{W}$ and $\mathcal{X}_{W}$ are equipped with the norms

$$
\left\|\left[z_{z_{2}}^{z_{1}}\right]\right\|_{\mathcal{Z}_{W}}^{2}:=\left\|z_{1}\right\|_{H^{2}(0,1)}^{2}+\left\|z_{2}\right\|_{H^{1}(0,1)}^{2} \quad \text { and } \quad\left\|\left[z_{z_{2}}^{z_{1}}\right]\right\|_{H^{1}(0,1) \times L^{2}(0,1)}^{2}:=\left\|z_{1}\right\|_{H^{1}(0,1)}^{2}+\left\|z_{2}\right\|_{L^{2}(0,1)}^{2},
$$

respectively. We will use the energy norm on $\mathcal{X}_{W}$, which for any $\rho>0$ is defined by

$$
\left\|\left[\begin{array}{c}
z_{1} \\
z_{2}
\end{array}\right]\right\|_{\mathcal{X}_{W}}^{2}:=\frac{1}{2}\left(\rho \int_{0}^{1}\left|z_{1}^{\prime}(s)\right|^{2} A(s) \mathrm{d} s+\frac{1}{\rho c^{2}} \int_{0}^{1}\left|z_{2}(s)\right|^{2} A(s) \Sigma(s)^{-2} \mathrm{~d} s\right) .
$$

This is an equivalent norm for $\mathcal{X}_{W}$ because the conditions (4.3) hold and $\sqrt{2}\left\|z_{1}\right\|_{L^{2}(0,1)} \leq\left\|z_{1}^{\prime}\right\|_{L^{2}(0,1)}$ for all $z_{1} \in H_{\{1\}}^{1}(0,1)$. To see that the Poincaré inequality holds in $H_{\{1\}}^{1}(0,1)$, note that for smooth functions $z$ with $z(1)=0$, one has from the fundamental theorem of calculus that

$$
|z(s)|=\left|\int_{s}^{1} z^{\prime}(t) \mathrm{d} t\right| \leq(1-s)^{1 / 2}\left\|z^{\prime}\right\|_{L^{2}(0,1)} .
$$


From this, we proceed by squaring and integrating with respect to $s$, and then passing to general Sobolev functions by approximation.

We define $\mathcal{U}_{W}:=\mathbb{C}$ with the absolute value norm $\left\|u_{0}\right\|_{\mathcal{U}_{W}}:=\left|u_{0}\right|$. The endpoint control and observation functionals $G_{W}: \mathcal{Z}_{W} \rightarrow \mathcal{U}_{W}$ and $K_{W}: \mathcal{Z}_{W} \rightarrow \mathcal{U}_{W}$ are defined by

$$
\begin{aligned}
& G_{W}\left[\begin{array}{l}
z_{1} \\
z_{2}
\end{array}\right]:=\frac{1}{2} \sqrt{\frac{A(0)}{\rho c(0)}}\left(-\rho c(0) z_{1}^{\prime}(0)+z_{2}(0)\right) \quad \text { and } \\
& K_{W}\left[\begin{array}{l}
z_{1} \\
z_{2}
\end{array}\right]:=\frac{1}{2} \sqrt{\frac{A(0)}{\rho c(0)}}\left(-\rho c(0) z_{1}^{\prime}(0)-z_{2}(0)\right) .
\end{aligned}
$$

Now the generalised Webster's horn model (4.1), (4.2) for the state $z(t)=\left[\begin{array}{l}\psi(t) \\ \pi(t)\end{array}\right]$ takes the form

$$
\left\{\begin{array}{l}
\frac{\mathrm{d}}{\mathrm{d} t}\left[\begin{array}{l}
\psi(t) \\
\pi(t)
\end{array}\right]=\left(L_{W}+\alpha H_{W}\right)\left[\begin{array}{l}
\psi(t) \\
\pi(t)
\end{array}\right], \\
\tilde{u}(t)=G_{W}\left[\begin{array}{l}
\psi(t) \\
\pi(t)
\end{array}\right]
\end{array}\right.
$$

and

$$
\tilde{y}(t)=K_{W}\left[\begin{array}{c}
\psi(t) \\
\pi(t)
\end{array}\right]
$$

for all $t \in \overline{\mathbb{R}}^{+}$. The initial conditions are $\left[\begin{array}{c}\psi(0) \\ \pi(0)\end{array}\right]=\left[\begin{array}{c}\psi_{0} \\ \pi_{0}\end{array}\right]$. The state variable $\pi=\rho \psi_{t}$ has the dimension of pressure, as in the case of the wave equation.

The impedance passive version of the following Theorem 4.1 is given in ([2], Thm. 5.1), and it would be possible to deduce parts of Theorem 4.1 from that result using the external Cayley transform ([30], Def. 3.1). Here we give a direct proof instead.

Theorem 4.1. Let the operators $L_{W}, H_{W}, G_{W}, K_{W}$, and spaces $\mathcal{Z}_{W}, \mathcal{X}_{W}, \mathcal{U}_{W}$ be defined as above. Let $\left[\begin{array}{l}\psi_{0} \\ \pi_{0}\end{array}\right] \in \mathcal{Z}_{W}$ and $\tilde{u} \in C^{2}\left(\overline{\mathbb{R}}^{+} ; \mathbb{C}\right)$ such that the compatibility condition $G_{W}\left[\begin{array}{l}\psi_{0} \\ \pi_{0}\end{array}\right]=\tilde{u}(0)$ holds. Then for all $\alpha \geq 0$ the following holds:

(i) The triple $\Xi_{\alpha}^{(W)}:=\left(G_{W}, L_{W}+\alpha H_{W}, K_{W}\right)$ is a scattering passive, strong boundary node on Hilbert spaces $\left(\mathcal{U}_{W}, \mathcal{X}_{W}, \mathcal{U}_{W}\right)$.

The semigroup generator $A_{W, \alpha}=\left.\left(L_{W}+\alpha H_{W}\right)\right|_{\operatorname{ker}\left(G_{W}\right)}$ of $\Xi_{\alpha}^{(W)}$ satisfies $A_{W, \alpha}^{*}=\left.\left(-L_{W}+\alpha H_{W}\right)\right|_{\operatorname{ker}\left(K_{W}\right)}$ and $0 \in \rho\left(A_{W, \alpha}\right) \cap \rho\left(A_{W, \alpha}^{*}\right)$.

(ii) The equations in (4.6) have a unique solution $\left[{ }_{\pi}^{\psi}\right] \in C^{1}\left(\overline{\mathbb{R}}^{+} ; \mathcal{X}_{W}\right) \cap C\left(\overline{\mathbb{R}}^{+} ; \mathcal{Z}_{W}\right)$. Hence we can define $\tilde{y} \in C\left(\overline{\mathbb{R}}^{+} ; \mathbb{C}\right)$ by equation $(4.7)$.

(iii) The solution of (4.6), (4.7) satisfies the energy dissipation inequality

$$
\frac{\mathrm{d}}{\mathrm{d} t}\left\|\left[\begin{array}{c}
\psi(t) \\
\pi(t)
\end{array}\right]\right\|_{\mathcal{X}_{W}}^{2} \leq|\tilde{u}(t)|^{2}-|\tilde{y}(t)|^{2}, \quad t \in \mathbb{R}^{+}
$$

Moreover, $\Xi_{0}^{(W)}$ is a conservative boundary node, and (4.8) holds then as an equality.

Under the assumptions of this proposition, we have $\psi \in C\left(\overline{\mathbb{R}}^{+} ; H^{2}(0,1)\right) \cap C^{1}\left(\overline{\mathbb{R}}^{+} ; H^{1}(0,1)\right) \cap C^{2}\left(\overline{\mathbb{R}}^{+} ; L^{2}(0,1)\right)$.

Proof. Claim (i): By Theorem 3.2, it is enough to show the conservative case $\alpha=0$. Let us first verify the that the Green-Lagrange identity

$$
\left.2 \operatorname{Re}\left\langle\left[\begin{array}{c}
z_{1} \\
z_{2}
\end{array}\right], L_{W}\left[\begin{array}{c}
z_{1} \\
z_{2}
\end{array}\right]\right\rangle\right\rangle_{W}+\left|K_{W}\left[\begin{array}{c}
z_{1} \\
z_{2}
\end{array}\right]\right|^{2}=\left|G_{W}\left[\begin{array}{c}
z_{1} \\
z_{2}
\end{array}\right]\right|^{2}
$$


holds for all $\left[\begin{array}{c}z_{1} \\ z_{2}\end{array}\right] \in \mathcal{Z}_{W}$. By partial integration, we get

$$
2 \operatorname{Re}\left\langle\left[\begin{array}{l}
z_{1} \\
z_{2}
\end{array}\right], L_{W}\left[\begin{array}{l}
z_{1} \\
z_{2}
\end{array}\right]\right\rangle_{\mathcal{X}_{W}}=-A(0) \operatorname{Re}\left(\overline{z_{1}^{\prime}(0)} z_{2}(0)\right)
$$

Now (4.9) follows since $\left|G_{W}\left[\begin{array}{c}z_{1} \\ z_{2}\end{array}\right]\right|^{2}-\left|K_{W}\left[\begin{array}{c}z_{1} \\ z_{2}\end{array}\right]\right|^{2}=-A(0) \operatorname{Re}\left(\overline{z_{1}^{\prime}(0)} z_{2}(0)\right)$ just as in equations (5.14), (5.15).

It is trivial that $G_{W} \mathcal{Z}_{W}=K_{W} \mathcal{Z}_{W}=\mathcal{U}_{W}$ since $\operatorname{dim} \mathcal{U}_{W}=1$ and neither of the operators $G_{W}$ and $K_{W}$ vanishes. We prove next that $L_{W}$ maps $\operatorname{ker}\left(G_{W}\right)$ bijectively onto $\mathcal{X}_{W}$. Now, $\left[\begin{array}{c}z_{1} \\ z_{2}\end{array}\right] \in \operatorname{ker}\left(G_{W}\right)$ and $\left[\begin{array}{c}w_{1} \\ w_{2}\end{array}\right] \in \mathcal{X}_{W}$ satisfy $L_{W}\left[\begin{array}{l}z_{1} \\ z_{2}\end{array}\right]=\left[\begin{array}{c}w_{1} \\ w_{2}\end{array}\right]$ if and only if $z_{2}=\rho w_{1}$ and

$$
\frac{\partial}{\partial s}\left(A(\cdot) \frac{\partial z_{1}}{\partial s}\right)=\frac{A(\cdot) w_{2}}{\rho c(\cdot)^{2}}, \quad z_{1}(1)=0, \quad z_{1}^{\prime}(0)=\frac{w_{1}(0)}{c(0)} .
$$

Since this equation has always a unique solution $z_{1} \in H^{2}(0,1)$ for any $w_{1} \in H_{\{1\}}^{1}(0,1)$ and $w_{2} \in L^{2}(0,1)$, it follows that $L_{W} \operatorname{ker}\left(G_{W}\right)=\mathcal{X}_{W}$ and $0 \in \rho\left(A_{W, 0}\right)$ where $A_{W, 0}=\left.L_{W}\right|_{\operatorname{ker}\left(G_{W}\right)}$ is the semigroup generator of $\Xi_{0}^{(W)}$. We conclude by Proposition 2.4 that $\Xi_{0}^{(W)}$ is a conservative boundary node as claimed. That $\Xi_{\alpha}^{(W)}$ is passive for $\alpha>0$ with semigroup generator $A_{W, \alpha}=\left.\left(L_{W}+\alpha H_{W}\right)\right|_{\operatorname{ker}\left(G_{W}\right)}$ follows by Theorem 3.2.

Because $H_{W}^{*}=H_{W} \in \mathcal{L}(\mathcal{X})$ is dissipative, we may apply Theorem 3.2 again to the time-flow inverted, conservative node $\left(\Xi_{0}^{(W)}\right)^{\leftarrow}=\left(K_{W},-L_{W}, G_{W}\right)$ to conclude that the boundary node $\left(K_{W},-L_{W}+\alpha H_{W}^{*}, G_{W}\right)$ is passive as well. Claim (iii) of Proposition 3.3 implies that $A_{W, \alpha}^{*}=\left.\left(-L_{W}+\alpha H_{W}\right)\right|_{\operatorname{ker}\left(K_{W}\right)}$.

Let us argue next that $0 \in \rho\left(A_{W, \alpha}\right) \cap \rho\left(A_{W, \alpha}^{*}\right)$ for $\alpha>0$. Because $A_{W, \alpha}$ is a compact resolvent operator, it is enough to exclude $0 \in \sigma_{p}\left(A_{W, \alpha}\right)$. Suppose $A_{W, \alpha} z_{0}=0$, giving $\operatorname{Re}\left\langle A_{W, 0} z_{0}, z_{0}\right\rangle_{\mathcal{X}}+\operatorname{Re}\left\langle\alpha H_{W} z_{0}, z_{0}\right\rangle_{\mathcal{X}}=$ $\operatorname{Re}\left\langle A_{W, \alpha} z_{0}, z_{0}\right\rangle_{\mathcal{X}}=0$. Thus

$$
\operatorname{Re}\left\langle A_{W, 0} z_{0}, z_{0}\right\rangle_{\mathcal{X}}=\alpha \operatorname{Re}\left\langle-H_{W} z_{0}, z_{0}\right\rangle_{\mathcal{X}}=\alpha\left\|\left(-H_{W}\right)^{1 / 2} z_{0}\right\|_{\mathcal{X}}^{2}=0
$$

by the dissipativity of both $A_{W, 0}$ and $H_{W}$, and the fact that $-H_{W}$ is a self-adjoint nonnegative operator. Thus $z_{0} \in \operatorname{ker}\left(H_{W}\right)$ and hence $A_{W, 0} z_{0}=\left(A_{W, 0}+\alpha H_{W}\right) z_{0}=A_{W, \alpha} z_{0}=0$. Because $0 \in \rho\left(A_{W, 0}\right)$ has already been shown, we conclude that $z_{0}=0$.

The node $\Xi_{0}^{(W)}$ is strong (i.e., $L_{W}$ is closed with $\left.\operatorname{dom}\left(L_{W}\right)=\mathcal{Z}_{W}\right)$ since $L_{W}=L_{W}^{* *}$. Indeed, we have $L_{W}^{*}=-\left.L_{W}\right|_{\operatorname{dom}\left(L_{W}^{*}\right)}$ where

$$
\operatorname{dom}\left(L_{W}^{*}\right)=\left\{\left[\begin{array}{c}
w_{1} \\
w_{2}
\end{array}\right] \in H_{\{1\}}^{1}(0,1) \cap H^{2}(0,1) \times H_{0}^{1}(0,1): \frac{\partial w_{1}}{\partial s}(0)=0\right\}
$$

which is dense in $\mathcal{X}_{W}$ and satisfies $\operatorname{dom}\left(L_{W}^{*}\right) \subset \operatorname{dom}\left(L_{W}\right)$. That $\Xi_{\alpha}^{(W)}$ is strong for $\alpha>0$ follows from $H_{W} \in \mathcal{L}(\mathcal{X})$ as explained in Theorem 3.2 .

Claims (ii) and (iii) follow from Proposition 2.2 and equation (2.4).

\section{Passive WAVE EQUATION ON WAVE GUIDES}

Define the tubular domain $\Omega \subset \mathbb{R}^{3}$ and its boundary components $\Gamma, \Gamma(0)$, and $\Gamma(1)$ as in Section 1 . Each of the sets $\Gamma, \Gamma(0)$, and $\Gamma(1)$ are smooth manifolds but $\partial \Omega=\bar{\Gamma} \cup \Gamma(0) \cup \Gamma(1)$ is only Lipschitz. Other relevant properties of $\Omega$ and $\partial \Omega$ are listed in (i)-(iii) of Appendix A where we also make rigorous sense of the Sobolev spaces, boundary trace mappings, Poincaré inequality, and the Green's identity for such domains. 
Following ([26], Sect. 3) we consider the linear dynamical system described by

$$
\left\{\begin{array}{l}
\phi_{t t}(\mathbf{r}, t)=c^{2} \Delta \phi(\mathbf{r}, t) \quad \text { for } \mathbf{r} \in \Omega \text { and } t \in \mathbb{R}^{+}, \\
c \frac{\partial \phi}{\partial \nu}(\mathbf{r}, t)+\phi_{t}(\mathbf{r}, t)=2 \sqrt{\frac{c}{\rho A(0)}} u(\mathbf{r}, t) \quad \text { for } \mathbf{r} \in \Gamma(0) \text { and } t \in \mathbb{R}^{+}, \\
\phi(\mathbf{r}, t)=0 \quad \text { for } \mathbf{r} \in \Gamma(1) \text { and } t \in \mathbb{R}^{+}, \\
\frac{\partial \phi}{\partial \nu}(\mathbf{r}, t)+\alpha \phi_{t}(\mathbf{r}, t)=0 \quad \text { for } \mathbf{r} \in \Gamma, \text { and } t \in \mathbb{R}^{+}, \text {and } \\
\phi(\mathbf{r}, 0)=\phi_{0}(\mathbf{r}), \quad \rho \phi_{t}(\mathbf{r}, 0)=p_{0}(\mathbf{r}) \quad \text { for } \mathbf{r} \in \Omega,
\end{array}\right.
$$

together with the observation $y$ defined by

$$
c \frac{\partial \phi}{\partial \nu}(\mathbf{r}, t)-\phi_{t}(\mathbf{r}, t)=2 \sqrt{\frac{c}{\rho A(0)}} y(\mathbf{r}, t) \quad \text { for } \mathbf{r} \in \Gamma(0) \text { and } t \in \mathbb{R}^{+} .
$$

This model describes acoustics of a cavity $\Omega$ that has an open end at $\Gamma(1)$ and an energy dissipating wall $\Gamma$. The solution $\phi$ is the velocity potential as its gradient is the perturbation velocity field of the acoustic waves. The boundary control and observation on surface $\Gamma(0)$ (whose area is $A(0)$ ) are both of scattering type. The speed of sound is denoted by $c>0$. The constants $\alpha \geq 0$ and $\rho>0$ have physical meaning but we refer to [26] for details. Note that if $\alpha=0$, we have the Neumann boundary condition modelling a hard, sound reflecting boundary on $\Gamma$. Our purpose is to show that (5.1), (5.2) defines a passive boundary node (conservative, if $\alpha=0$ by a slightly different argument in Cor. 5.2) by using Theorem 3.1 with the aid of the additional signals $\tilde{u}:=\frac{1}{\sqrt{\alpha}} \frac{\partial \phi}{\partial \nu}+\sqrt{\alpha} \phi_{t}$ (that will be grounded) and $\tilde{y}:=\frac{1}{\sqrt{\alpha}} \frac{\partial \phi}{\partial \nu}-\sqrt{\alpha} \phi_{t}$ (that will be disregarded) on the wall $\Gamma$.

The boundedness of the Dirichlet trace implies that the space

$$
H_{\Gamma(1)}^{1}(\Omega):=\left\{f \in H^{1}(\Omega):\left.f\right|_{\Gamma(1)}=0\right\} .
$$

is a closed subspace of $H^{1}(\Omega)$. Define

$$
\tilde{\mathcal{Z}}^{\prime}:=\left\{f \in H_{\Gamma(1)}^{1}(\Omega): \Delta f \in L^{2}(\Omega),\left.\frac{\partial f}{\partial \nu}\right|_{\Gamma(0) \cup \Gamma} \in L^{2}(\Gamma(0) \cup \Gamma)\right\}
$$

with the norm $\|f\|_{\tilde{\mathcal{Z}}^{\prime}}^{2}=\|f\|_{H^{1}(\Omega)}^{2}+\|\Delta f\|_{L^{2}(\Omega)}^{2}+\left\|\left.\frac{\partial f}{\partial \nu}\right|_{\Gamma(0) \cup \Gamma}\right\|_{L^{2}(\Gamma(0) \cup \Gamma)}^{2}$. Then the operator

$$
\left.\frac{\partial}{\partial \nu}\right|_{\Gamma^{\prime}}:\left.f \mapsto \frac{\partial f}{\partial \nu}\right|_{\Gamma^{\prime}} \quad \text { lies in } \quad \mathcal{L}\left(\tilde{\mathcal{Z}}^{\prime} ; L^{2}\left(\Gamma^{\prime}\right)\right) \quad \text { for } \quad \Gamma^{\prime} \in\{\Gamma(0), \Gamma, \Gamma(0) \cup \Gamma\}
$$

The spaces $\tilde{\mathcal{Z}}, \mathcal{X}$, and the interior operator $L$ are defined by

$$
\begin{gathered}
L:=\left[\begin{array}{cc}
0 & \rho^{-1} \\
\rho c^{2} \Delta & 0
\end{array}\right]: \tilde{\mathcal{Z}} \rightarrow \mathcal{X} \quad \text { with } \\
\tilde{\mathcal{Z}}:=\tilde{\mathcal{Z}}^{\prime} \times H_{\Gamma(1)}^{1}(\Omega) \quad \text { and } \quad \mathcal{X}:=H_{\Gamma(1)}^{1}(\Omega) \times L^{2}(\Omega)
\end{gathered}
$$

where $H_{\Gamma(1)}^{1}(\Omega)$ and $\tilde{\mathcal{Z}}^{\prime}$ are given by $(5.3),(5.4)$. For the space $\mathcal{X}$, we use the energy norm, which is defined by

$$
\left\|\left[\begin{array}{c}
z_{1} \\
z_{2}
\end{array}\right]\right\|_{\mathcal{X}}^{2}:=\frac{1}{2}\left(\rho\left\|\mid \nabla z_{1}\right\|_{L^{2}(\Omega)}^{2}+\frac{1}{\rho c^{2}}\left\|z_{2}\right\|_{L^{2}(\Omega)}^{2}\right) .
$$

The Poincaré inequality $\left\|z_{1}\right\|_{L^{2}(\Omega)} \leq M_{\Omega}\left\|\nabla z_{1}\right\|_{L^{2}(\Omega)}$ holds for $z_{1} \in H_{\Gamma(1)}^{1}(\Omega)$ as given in Theorem A.4 in Appendix A. Therefore (5.7) defines a norm on $\mathcal{X}$, equivalent to the Cartesian product norm

$$
\left\|\left[\begin{array}{c}
z_{1} \\
z_{2}
\end{array}\right]\right\|_{H^{1}(\Omega) \times L^{2}(\Omega)}^{2}:=\left\|z_{1}\right\|_{L^{2}(\Omega)}^{2}+\left\|\nabla z_{1}\right\|_{L^{2}(\Omega)}^{2}+\left\|z_{2}\right\|_{L^{2}(\Omega)}^{2}
$$


so that $\tilde{\mathcal{Z}} \subset \mathcal{X}$ with a continuous embedding, and $L \in \mathcal{L}(\tilde{\mathcal{Z}} ; \mathcal{X})$ with respect to the $\tilde{\mathcal{Z}}$-norm

$$
\left\|\left[\begin{array}{c}
z_{1} \\
z_{2}
\end{array}\right]\right\|_{\tilde{\mathcal{Z}}}^{2}:=\left\|z_{1}\right\|_{\tilde{\mathcal{Z}}^{\prime}}^{2}+\left\|z_{2}\right\|_{L^{2}(\Omega)}^{2}+\left\|\nabla z_{2}\right\|_{L^{2}(\Omega)}^{2} .
$$

Defining $\mathcal{U}:=L^{2}(\Gamma(0))$ and $\tilde{\mathcal{U}}:=L^{2}(\Gamma)$ with the norms

$$
\left\|u_{0}\right\|_{\mathcal{U}}^{2}=A(0)^{-1}\left\|u_{0}\right\|_{L^{2}(\Gamma(0))}^{2} \text { and }\left\|\tilde{u}_{0}\right\|_{\tilde{\mathcal{U}}}=\left\|\tilde{u}_{0}\right\|_{L^{2}(\Gamma)},
$$

we get $\mathcal{U} \oplus \tilde{\mathcal{U}}=L^{2}(\Gamma(0) \cup \Gamma)$ where we use the Cartesian product norm of $\mathcal{U}$ and $\tilde{\mathcal{U}}$.

The boundedness of the Dirichlet trace and the property (5.5) of the Neumann trace imply that $\left[\begin{array}{c}G \\ G_{\alpha}\end{array}\right] \in$ $\mathcal{L}(\tilde{\mathcal{Z}} ; \mathcal{U} \oplus \tilde{\mathcal{U}})$ and $\left[\begin{array}{c}K \\ K_{\alpha}\end{array}\right] \in \mathcal{L}(\tilde{\mathcal{Z}} ; \mathcal{U} \oplus \tilde{\mathcal{U}})$ where

$$
\begin{aligned}
& {\left[\begin{array}{c}
G \\
G_{\alpha}
\end{array}\right]\left[\begin{array}{c}
z_{1} \\
z_{2}
\end{array}\right]:=\frac{1}{2}\left[\begin{array}{c}
\sqrt{\frac{A(0)}{\rho c}}\left(\left.\rho c \frac{\partial z_{1}}{\partial \nu}\right|_{\Gamma(0)}+\left.z_{2}\right|_{\Gamma(0)}\right) \\
\left.\frac{\sqrt{\rho}}{\sqrt{\alpha}} \frac{\partial z_{1}}{\partial \nu}\right|_{\Gamma}+\left.\frac{\sqrt{\alpha}}{\sqrt{\rho}} z_{2}\right|_{\Gamma}
\end{array}\right] \quad \text { and }} \\
& {\left[\begin{array}{c}
K \\
K_{\alpha}
\end{array}\right]\left[\begin{array}{c}
z_{1} \\
z_{2}
\end{array}\right]:=\frac{1}{2}\left[\begin{array}{c}
\sqrt{\frac{A(0)}{\rho c}}\left(\left.\rho c \frac{\partial z_{1}}{\partial \nu}\right|_{\Gamma(0)}-\left.z_{2}\right|_{\Gamma(0)}\right) \\
\left.\frac{\sqrt{\rho}}{\sqrt{\alpha}} \frac{\partial z_{1}}{\partial \nu}\right|_{\Gamma}-\left.\frac{\sqrt{\alpha}}{\sqrt{\rho}} z_{2}\right|_{\Gamma}
\end{array}\right]}
\end{aligned}
$$

The reason for defining the triple $\widetilde{\Xi}_{\alpha}:=\left(\left[\begin{array}{c}G \\ G_{\alpha}\end{array}\right], L,\left[\begin{array}{c}K \\ K_{\alpha}\end{array}\right]\right)$ is to obtain first order equations from (5.1), using the equivalence of $\phi_{t t}=c^{2} \Delta \phi$ and $\frac{\mathrm{d}}{\mathrm{d} t}\left[\begin{array}{l}\phi \\ p\end{array}\right]=\left[\begin{array}{cc}0 & \rho^{-1} \\ \rho c^{2} \Delta & 0\end{array}\right]\left[\begin{array}{l}\phi \\ p\end{array}\right]$ where $p=\rho \phi_{t}$ is the sound pressure. More precisely, equations (5.1), (5.2) are (at least formally) equivalent with

$$
\left\{\begin{array}{c}
\frac{\mathrm{d}}{\mathrm{d} t}\left[\begin{array}{l}
\phi(t) \\
p(t)
\end{array}\right]=L\left[\begin{array}{l}
\phi(t) \\
p(t)
\end{array}\right] \\
{\left[\begin{array}{c}
u(t) \\
0
\end{array}\right]=\left[\begin{array}{c}
G \\
G_{\alpha}
\end{array}\right]\left[\begin{array}{l}
\phi(t) \\
p(t)
\end{array}\right]}
\end{array}\right.
$$

and

$$
\left[\begin{array}{l}
y(t) \\
\tilde{y}(t)
\end{array}\right]=\left[\begin{array}{c}
K \\
K_{\alpha}
\end{array}\right]\left[\begin{array}{l}
\phi(t) \\
p(t)
\end{array}\right]
$$

for $t \in \overline{\mathbb{R}}^{+}$, with the initial conditions $\left[\begin{array}{l}\phi(0) \\ p(0)\end{array}\right]=\left[\begin{array}{l}\phi_{0} \\ p_{0}\end{array}\right]$. The Green-Lagrange identity

$$
2 \operatorname{Re}\left\langle\left[\begin{array}{c}
z_{1} \\
z_{2}
\end{array}\right], L\left[\begin{array}{c}
z_{1} \\
z_{2}
\end{array}\right]\right\rangle_{\mathcal{X}}+\left\|\left[\begin{array}{c}
K \\
K_{\alpha}
\end{array}\right]\left[\begin{array}{c}
z_{1} \\
z_{2}
\end{array}\right]\right\|_{\mathcal{U} \oplus \tilde{\mathcal{U}}}^{2}=\left\|\left[\begin{array}{c}
G \\
G_{\alpha}
\end{array}\right]\left[\begin{array}{c}
z_{1} \\
z_{2}
\end{array}\right]\right\|_{\mathcal{U} \oplus \tilde{\mathcal{U}}}^{2} \text { for all }\left[\begin{array}{c}
z_{1} \\
z_{2}
\end{array}\right] \in \tilde{\mathcal{Z}}
$$

is a key fact for proving the conservativity of $\widetilde{\Xi}_{\alpha}$, and we verify it next. Green's identity (Thm. A.3 in Appendix A) gives

$$
\begin{aligned}
& 2 \operatorname{Re}\left\langle\left[\begin{array}{c}
z_{1} \\
z_{2}
\end{array}\right], L\left[\begin{array}{c}
z_{1} \\
z_{2}
\end{array}\right]\right\rangle_{\mathcal{X}}=2 \operatorname{Re}\left\langle\left[\begin{array}{c}
z_{1} \\
z_{2}
\end{array}\right],\left[\begin{array}{c}
\rho^{-1} z_{2} \\
\rho c^{2} \Delta z_{1}
\end{array}\right]\right\rangle_{\mathcal{X}} \\
& =2 \operatorname{Re} \frac{1}{2}\left(\rho \int_{\Omega} \nabla \overline{z_{1}} \cdot \nabla\left(z_{2} / \rho\right) \mathrm{d} V+\frac{1}{\rho c^{2}}\left\langle\rho c^{2} \Delta z_{1}, z_{2}\right\rangle_{L^{2}(\Omega)}\right) \\
& =\operatorname{Re}\left(\int_{\Gamma(0) \cup \Gamma \cup \Gamma(1)} \frac{\partial \overline{z_{1}}}{\partial \nu} z_{2} \mathrm{~d} A\right) \\
& =\operatorname{Re}\left\langle\left.\frac{\partial z_{1}}{\partial \nu}\right|_{\Gamma(0)},\left.z_{2}\right|_{\Gamma(0)}\right\rangle_{L^{2}(\Gamma(0))}+\operatorname{Re}\left\langle\left.\frac{\partial z_{1}}{\partial \nu}\right|_{\Gamma},\left.z_{2}\right|_{\Gamma}\right\rangle_{L^{2}(\Gamma)}
\end{aligned}
$$


because $\left.z_{2}\right|_{\Gamma(1)}=0$ by (5.6). On the other hand, we obtain

$$
\begin{aligned}
& \left\|G\left[\begin{array}{c}
z_{1} \\
z_{2}
\end{array}\right]\right\|_{\mathcal{U}}^{2}=A(0)^{-1}\left\langle G\left[\begin{array}{c}
z_{1} \\
z_{2}
\end{array}\right], G\left[\begin{array}{l}
z_{1} \\
z_{2}
\end{array}\right]\right\rangle_{L^{2}(\Gamma(0))} \\
& =\frac{1}{4 \rho c}\left(\rho^{2} c^{2}\left\|\left.\frac{\partial z_{1}}{\partial \nu}\right|_{\Gamma(0)}\right\|_{L^{2}(\Gamma(0))}^{2}+2 \rho c \operatorname{Re}\left\langle\left.\frac{\partial z_{1}}{\partial \nu}\right|_{\Gamma(0)},\left.z_{2}\right|_{\Gamma(0)}\right\rangle_{L^{2}(\Gamma(0))}+\left\|\left.z_{2}\right|_{\Gamma(0)}\right\|_{L^{2}(\Gamma(0))}^{2}\right)
\end{aligned}
$$

and also

$$
\begin{aligned}
& \left\|K\left[\begin{array}{l}
z_{1} \\
z_{2}
\end{array}\right]\right\|_{\mathcal{U}}^{2}=A(0)^{-1}\left\langle K\left[\begin{array}{c}
z_{1} \\
z_{2}
\end{array}\right], K\left[\begin{array}{c}
z_{1} \\
z_{2}
\end{array}\right]\right\rangle_{L^{2}(\Gamma(0))} \\
& =\frac{1}{4 \rho c}\left(\rho^{2} c^{2}\left\|\left.\frac{\partial z_{1}}{\partial \nu}\right|_{\Gamma(0)}\right\|_{L^{2}(\Gamma(0))}^{2}-2 \rho c \operatorname{Re}\left\langle\left.\frac{\partial z_{1}}{\partial \nu}\right|_{\Gamma(0)},\left.z_{2}\right|_{\Gamma(0)}\right\rangle_{L^{2}(\Gamma(0))}+\left\|\left.z_{2}\right|_{\Gamma(0)}\right\|_{L^{2}(\Gamma(0))}^{2}\right),
\end{aligned}
$$

where $G\left[\begin{array}{l}z_{1} \\ z_{2}\end{array}\right]$ and $K\left[\begin{array}{l}z_{1} \\ z_{2}\end{array}\right]$ are the first components in (5.9) respectively.

Similarly, we compute the two terms needed in

$$
\left\|G_{\alpha}\left[\begin{array}{c}
z_{1} \\
z_{2}
\end{array}\right]\right\|_{\tilde{\mathcal{U}}}^{2}-\left\|K_{\alpha}\left[\begin{array}{c}
z_{1} \\
z_{2}
\end{array}\right]\right\|_{\tilde{\mathcal{U}}}^{2}=\left\langle G_{\alpha}\left[\begin{array}{c}
z_{1} \\
z_{2}
\end{array}\right], G_{\alpha}\left[\begin{array}{c}
z_{1} \\
z_{2}
\end{array}\right]\right\rangle_{L^{2}(\Gamma)}-\left\langle K_{\alpha}\left[\begin{array}{c}
z_{1} \\
z_{2}
\end{array}\right], K_{\alpha}\left[\begin{array}{c}
z_{1} \\
z_{2}
\end{array}\right]\right\rangle_{L^{2}(\Gamma)}=\operatorname{Re}\left\langle\left.\frac{\partial z_{1}}{\partial \nu}\right|_{\Gamma},\left.z_{2}\right|_{\Gamma}\right\rangle_{L^{2}(\Gamma)}
$$

where $G_{\alpha}\left[\begin{array}{c}z_{1} \\ z_{2}\end{array}\right]$ and $K_{\alpha}\left[\begin{array}{c}z_{1} \\ z_{2}\end{array}\right]$ are the second components in (5.9) respectively. Now (5.13), (5.16) implies (5.12) as required.

We proceed to show that the the triple $\Xi_{\alpha}:=\left(\left.G\right|_{\mathcal{Z}_{\alpha}},\left.L\right|_{\mathcal{Z}_{\alpha}},\left.K\right|_{\mathcal{Z}_{\alpha}}\right)$ for all $\alpha>0$ is a scattering passive boundary node on Hilbert spaces $(\mathcal{U}, \mathcal{X}, \mathcal{U})$ with the solution space

$$
\mathcal{Z}_{\alpha}:=\left\{\left[\begin{array}{c}
z_{1} \\
z_{2}
\end{array}\right] \in \tilde{\mathcal{Z}}^{\prime} \times H_{\Gamma(1)}^{1}(\Omega):\left.\frac{\partial z_{1}}{\partial \nu}\right|_{\Gamma}+\left.\frac{\alpha}{\rho} z_{2}\right|_{\Gamma}=0\right\} .
$$

Note that $\mathcal{Z}_{\alpha}$ is a closed subspace of $\tilde{\mathcal{Z}}$ because $G_{\alpha} \in \mathcal{L}(\tilde{\mathcal{Z}} ; \tilde{\mathcal{U}})$ and $\mathcal{Z}_{\alpha}=\operatorname{ker}\left(G_{\alpha}\right)$. Therefore, we can use the norm of $\tilde{\mathcal{Z}}$ on $\mathcal{Z}_{\alpha}$. The conservative case $\alpha=0$ is slightly different, and it is treated separately in Corollary 5.2.

Theorem 5.1. Take $\alpha>0$ and let the operators $L, G, K$, and Hilbert spaces $\mathcal{X}, \mathcal{U}$, and $\mathcal{Z}_{\alpha}$ be defined as above. Let $\left[\begin{array}{c}\phi_{0} \\ p_{0}\end{array}\right] \in \mathcal{Z}_{\alpha}$ and $u \in C^{2}\left(\overline{\mathbb{R}}^{+} ; \mathcal{U}\right)$ be such that the compatibility condition $G\left[\begin{array}{l}\phi_{0} \\ p_{0}\end{array}\right]=u(0)$ holds. Then the following hold:

(i) The triple $\Xi_{\alpha}:=\left(\left.G\right|_{\mathcal{Z}_{\alpha}},\left.L\right|_{\mathcal{Z}_{\alpha}},\left.K\right|_{\mathcal{Z}_{\alpha}}\right)$ is a scattering passive boundary node on Hilbert spaces $(\mathcal{U}, \mathcal{X}, \mathcal{U})$ with solution space $\mathcal{Z}_{\alpha}$. The semigroup generator $A_{\alpha}=\left.L\right|_{\operatorname{ker}(G) \cap \operatorname{ker}\left(G_{\alpha}\right)}$ of $\Xi_{\alpha}$ satisfies $A_{\alpha}^{*}=-\left.L\right|_{\operatorname{ker}(K) \cap \operatorname{ker}\left(K_{\alpha}\right)}$ and $0 \in \rho\left(A_{\alpha}\right) \cap \rho\left(A_{\alpha}^{*}\right)$.

(ii) The equations ${ }^{6}$ in $(5.10)$ have a unique solution $\left[\begin{array}{l}\phi \\ p\end{array}\right] \in C^{1}\left(\overline{\mathbb{R}}^{+} ; \mathcal{X}\right) \cap C\left(\overline{\mathbb{R}}^{+} ; \mathcal{Z}_{\alpha}\right)$ satisfying $\phi(0)=\phi_{0}$ and $p(0)=p_{0}$. Hence we can define $y \in C\left(\overline{\mathbb{R}}^{+} ; \mathcal{U}\right)$ by equation $(5.11)$.

(iii) The solutions of (5.10), (5.11) satisfy the energy dissipation inequality

$$
\frac{\mathrm{d}}{\mathrm{d} t}\left\|\left[\begin{array}{c}
\phi(t) \\
p(t)
\end{array}\right]\right\|_{\mathcal{X}}^{2} \leq\|u(t)\|_{\mathcal{U}}^{2}-\|y(t)\|_{\mathcal{U}}^{2}, \quad t \in \mathbb{R}^{+} .
$$

It follows from Claim (ii) and the definition of the norms of $\mathcal{Z}_{\alpha}$ and $\mathcal{X}$ that $\phi \in C^{1}\left(\overline{\mathbb{R}}^{+} ; H^{1}(\Omega)\right) \cap C^{2}\left(\overline{\mathbb{R}}^{+} ; L^{2}(\Omega)\right)$, $\nabla \phi \in C^{1}\left(\overline{\mathbb{R}}^{+} ; L^{2}\left(\Omega ; \mathbb{R}^{3}\right)\right)$, and $\Delta \phi \in C\left(\overline{\mathbb{R}}^{+} ; L^{2}(\Omega)\right)$. These are the same smoothness properties that have been used in ([26], see, in particular, Eq. (1.4)) for deriving the generalised Webster's equation in (1.3) from the wave equation.

\footnotetext{
${ }^{6}$ Note that (2.1) is equivalent with (5.1) and (5.10) in the context of this theorem.
} 
Proof.

Claim (i): By Theorem 3.1 and the discussion preceding this theorem, it is enough to show that $\widetilde{\Xi}_{\alpha}=$ $\left(\left[\begin{array}{c}G \\ G_{\alpha}\end{array}\right], L,\left[\begin{array}{c}K \\ K_{\alpha}\end{array}\right]\right)$ introduced above is a conservative boundary node which is easiest done by using Proposition 2.4. Since the Green-Lagrange identity (2.6) has already been established, it remains to prove conditions (ii) (with $\left[\begin{array}{c}G \\ G_{\alpha}\end{array}\right]$ in place of $G$ ) and (iv) (with $\left[\begin{array}{c}K \\ K_{\alpha}\end{array}\right]$ in place of $K$ ) of Proposition 2.4 with $\beta=\gamma=0$. It is enough to consider only $\beta=\gamma=0$ because the resolvent sets of $\left.L\right|_{\operatorname{ker}(G)}$ and $-\left.L\right|_{\operatorname{ker}(K)}$ in Proposition 2.4 are open, and then the same conditions hold for some $\beta, \gamma>0$ as well.

For an arbitrary $g \in L^{2}(\Gamma(0) \cup \Gamma)$ there exists a unique variational ${ }^{7}$ solution $z_{1} \in H_{\Gamma(1)}^{1}(\Omega)$ of the problem

$$
\Delta z_{1}=0,\left.\quad z_{1}\right|_{\Gamma(1)}=0,\left.\quad \frac{\partial z_{1}}{\partial \nu}\right|_{\Gamma(0) \cup \Gamma}=g .
$$

Since $z_{1} \in \tilde{\mathcal{Z}}^{\prime}$, we have $\left.\frac{\partial}{\partial \nu}\right|_{\Gamma(0) \cup \Gamma} \tilde{\mathcal{Z}}^{\prime}=L^{2}(\Gamma(0) \cup \Gamma)$ which obviously gives both $\left.\frac{\partial}{\partial \nu}\right|_{\Gamma(0)} \tilde{\mathcal{Z}}^{\prime}=L^{2}(\Gamma(0))$ and $\left.\frac{\partial}{\partial \nu}\right|_{\Gamma} \tilde{\mathcal{Z}}^{\prime}=L^{2}(\Gamma)$. Clearly $\tilde{\mathcal{Z}}^{\prime} \oplus\{0\} \subset \tilde{\mathcal{Z}}$ and the surjectivity of $\left[\begin{array}{c}G \\ G_{\alpha}\end{array}\right]$ follows from

$$
\left[\begin{array}{c}
G \\
G_{\alpha}
\end{array}\right]\left[\begin{array}{c}
z_{1} \\
0
\end{array}\right]:=\frac{1}{2}\left[\begin{array}{c}
\left.\sqrt{A(0) \rho c} \frac{\partial}{\partial \nu}\right|_{\Gamma(0)} \\
\left.\frac{\sqrt{\rho}}{\sqrt{\alpha}} \frac{\partial}{\partial \nu}\right|_{\Gamma}
\end{array}\right] z_{1} .
$$

To see this, for a given $h \in L^{2}(\Gamma(0) \cup \Gamma)$, we choose

$$
g= \begin{cases}2 \frac{1}{\sqrt{A(0) \rho c}} h, & \text { on } \Gamma(0), \\ 2 \frac{\sqrt{\alpha}}{\sqrt{\rho}} h, & \text { on } \Gamma\end{cases}
$$

in (5.19) to find a function $z_{1}$ so that $\left[\begin{array}{c}G \\ G_{\alpha}\end{array}\right]\left[\begin{array}{c}z_{1} \\ 0\end{array}\right]=h$. The surjectivity of $\left[\begin{array}{c}K \\ K_{\alpha}\end{array}\right]$ is proved similarly.

To show that $L \operatorname{ker}\left(\left[\begin{array}{c}G \\ G_{\alpha}\end{array}\right]\right)=L\left(\operatorname{ker}(G) \cap \operatorname{ker}\left(G_{\alpha}\right)\right)=\mathcal{X}$, let $\left[\begin{array}{c}w_{1} \\ w_{2}\end{array}\right] \in \mathcal{X}$ be arbitrary. Then $\left[\begin{array}{c}w_{1} \\ w_{2}\end{array}\right]=L\left[\begin{array}{c}z_{1} \\ z_{2}\end{array}\right]=$ $\left[\begin{array}{c}\rho^{-1} z_{2} \\ \rho c^{2} \Delta z_{1}\end{array}\right]$ for $\left[\begin{array}{c}z_{1} \\ z_{2}\end{array}\right] \in \operatorname{ker}(G) \cap \operatorname{ker}\left(G_{\alpha}\right)$ if and only if $z_{2}=\rho w_{1}$ and the variational solution $z_{1} \in H_{\Gamma(1)}^{1}(\Omega)$ of the problem

$$
\rho c^{2} \Delta z_{1}=w_{2},\left.\quad z_{1}\right|_{\Gamma(1)}=0,\left.\quad \frac{\partial z_{1}}{\partial \nu}\right|_{\Gamma}=-\left.\alpha \rho w_{1}\right|_{\Gamma},\left.\quad c \frac{\partial z_{1}}{\partial \nu}\right|_{\Gamma(0)}=-\left.w_{1}\right|_{\Gamma(0)}
$$

exists and belongs to the space $\mathcal{Z}^{\prime}$. This condition can be verified by standard variational techniques because $w_{2} \in L^{2}(\Omega)$ and $w_{1} \in H_{\Gamma(1)}^{1}(\Omega)$ which implies $\left.w_{1}\right|_{\Gamma(0) \cup \Gamma} \in H^{1 / 2}(\Gamma(0) \cup \Gamma) \subset L^{2}(\Gamma(0) \cup \Gamma)$. That $L \operatorname{ker}\left(\left[\begin{array}{c}K \\ K_{\alpha}\end{array}\right]\right)=$ $\mathcal{X}$ is proved similarly. All the conditions of Proposition 2.4 are now satisfied with $\beta=\gamma=0$, and thus $\tilde{\Xi}_{\alpha}$ is a conservative boundary node. It follows from Theorem 3.1 that $\Xi_{\alpha}$ is a passive boundary node which has the common semigroup generator $A_{\alpha}=\left.L\right|_{\operatorname{ker}(G) \cap \operatorname{ker}\left(G_{\alpha}\right)}$ with the original conservative boundary node $\tilde{\Xi}_{\alpha}$. By [29], Theorem 1.9 and Proposition 4.3, the dual system of $\widetilde{\Xi}_{\alpha}$ is of boundary control type, and it coincides with the time-flow inverted boundary node $\widetilde{\Xi}_{\alpha}^{\leftarrow}$. The unbounded adjoint $A_{\alpha}^{*}$ is the semigroup generator of the dual system $\widetilde{\Xi}_{\alpha}^{\leftarrow}$, and hence $A_{\alpha}^{*}=-\left.L\right|_{\operatorname{ker}(K) \cap \operatorname{ker}\left(K_{\alpha}\right)}$ as claimed.

It remains to show that $0 \notin \sigma\left(A_{\alpha}\right)$. We have already shown above that $A_{\alpha} \operatorname{dom}\left(A_{\alpha}\right)=\mathcal{X}$ with $\operatorname{dom}\left(A_{\alpha}\right)=$ $\operatorname{ker}(G) \cap \operatorname{ker}\left(G_{\alpha}\right)$, and the remaining injectivity part follows if we show that $\operatorname{ker}(L) \cap \operatorname{ker}(G) \cap \operatorname{ker}\left(G_{\alpha}\right)=\{0\}$. This follows because the variational solution in $H^{1}(\Omega)$ of the homogenous problem

$$
\Delta z_{1}=0,\left.\quad z_{1}\right|_{\Gamma(1)}=0,\left.\quad \frac{\partial z_{1}}{\partial \nu}\right|_{\Gamma(0) \cup \Gamma}=0
$$

is unique. That $0 \notin \sigma\left(A_{\alpha}^{*}\right)$ follows similarly by considering the time-flow inverted system $\widetilde{\Xi}_{\alpha}^{\leftarrow}$ instead.

Claims (ii), (iii): Since scattering passive boundary nodes are internally well-posed, it follows from (e.g., [29], Lem. 2.6) that equations (2.1) are solvable, as has been explained in Section 2.

\footnotetext{
${ }^{7}$ We leave it to the interested reader to derive the variational form using Green's identity (A.9) and then carry out the usual argument by the Lax-Milgram theorem; see (e.g., [12], Lem. 2.2.1.1).
} 
Corollary 5.2. Use the same notation and make the same assumptions as in Theorem 5.1. If $\alpha=0$, then Claims (i), (iii) of Theorem 5.1 hold in the stronger form: (i') the triple $\Xi_{0}:=\left(\left.G\right|_{\mathcal{Z}_{0}},\left.L\right|_{\mathcal{Z}_{0}},\left.K\right|_{\mathcal{Z}_{0}}\right)$ is a scattering conservative boundary node on Hilbert spaces $(\mathcal{U}, \mathcal{X}, \mathcal{U})$ with the solution space $\mathcal{Z}_{0}:=\tilde{\mathcal{Z}}_{0}^{\prime} \times H_{\Gamma(1)}^{1}(\Omega)$ where

$$
\tilde{\mathcal{Z}}_{0}^{\prime}:=\left\{f \in H_{\Gamma(1)}^{1}(\Omega): \Delta f \in L^{2}(\Omega),\left.\frac{\partial f}{\partial \nu}\right|_{\Gamma(0)} \in L^{2}(\Gamma(0)),\left.\frac{\partial f}{\partial \nu}\right|_{\Gamma}=0\right\} ;
$$

and (iii') the energy inequality (5.18) holds as an equality.

Claim (ii) of Theorem 5.1 remains true without change. Thus, the solution $\phi$ has the same regularity properties as listed right after Theorem 5.1.

Proof. Because the operators $G_{\alpha}$ and $K_{\alpha}$ refer to $1 / \sqrt{\alpha}$, we cannot simply set $\alpha=0$ in the proof. This problem could be resolved by making the norm of $\tilde{\mathcal{U}}$ dependent on $\alpha$ which we want to avoid. A direct argument can be given without ever defining $\widetilde{\Xi}_{\alpha}$. To prove the Green-Lagrange identity

$$
2 \operatorname{Re}\left\langle\left[\begin{array}{c}
z_{1} \\
z_{2}
\end{array}\right], L\left[\begin{array}{c}
z_{1} \\
z_{2}
\end{array}\right]\right\rangle \mathcal{X}+\left\|K\left[\begin{array}{c}
z_{1} \\
z_{2}
\end{array}\right]\right\|_{\mathcal{U}}^{2}=\left\|G\left[\begin{array}{c}
z_{1} \\
z_{2}
\end{array}\right]\right\| \|_{\mathcal{U}}^{2} \text { for all }\left[\begin{array}{c}
z_{1} \\
z_{2}
\end{array}\right] \in \tilde{\mathcal{Z}}_{0}
$$

for $\Xi_{0}$, one simply omits the last term on the right hand side of (5.13) by using the Neumann condition $\left.\frac{\partial z_{1}}{\partial \nu}\right|_{\Gamma}=0$ from (5.20). Then (5.21) follows from (5.13), (5.15), leading ultimately to (5.18) with an equality. The remaining parts of Claim (i') follow by the argument given in the proof of Theorem 5.1.

This result generalises the reflecting mirror example in ([29], Sect. 5) and further generalisations are given in Section 6.

\section{Conclusions And generalisations}

We have given a unified treatment of a 3D wave equation model on tubular structures and the corresponding Webster's horn model in the form it is derived and used in $[25,26]$. Both the forward time solvability and the energy inequalities have been treated rigorously, and the necessary but hard-to-find Sobolev space apparatus was presented in Appendix A. The strictly dissipative case was reduced to the conservative case using auxiliary Theorems 3.1 and 3.2 that have independent interest.

Theorem 5.1 can be extended and generalised significantly using only the techniques presented in this work. Firstly, a dissipation term, analogous with the one appearing in Webster's equation (4.1), can be added to the wave equation part of (5.1) while keeping rest of the model the same:

Corollary 6.1. Theorem 5.1 remains true if the wave equation $\phi_{t t}=c^{2} \Delta \phi$ in (5.1) is replaced by $\phi_{t t}=$ $c^{2} \Delta \phi+g(\cdot) \phi_{t}$ where $g$ is a smooth function satisfying $g(\mathbf{r}) \leq 0$ for all $\mathbf{r} \in \Omega$.

Indeed, this follows by using Theorem 3.2 on the result of Theorem 5.1 in the same way as has been done in Section 4. Even now the resulting negative perturbation $H$ on the original interior operator $L$ in (5.6) satisfies $H \in \mathcal{L}(\mathcal{X})$. The same dissipation term can, of course, be added to Corollary 5.2 (where $\alpha=0$ ) as well but then the resulting boundary node is only passive unless $g \equiv 0$.

Theorem 5.1 can be generalised to cover much more complicated geometries $\Omega \subset \mathbb{R}^{3}$ than tube segments with circular cross-sections. Inspecting the construction of the boundary node $\Xi_{\alpha}$ and the accompanying Hilbert spaces in Section 5, it becomes clear that much more can be proved at the cost of more complicated notation but nothing more:

Corollary 6.2. Let $\Omega \subset \mathbb{R}^{3}$ be a bounded Lipschitz domain satisfying standing assumptions (i)-(iv) in Appendix (i). Denote the smooth boundary components of $\Omega$ by $\Gamma_{j}$ where $j \in J \subset \mathbb{N}$ satisfying $\partial \Omega=\cup_{j \in J} \overline{\Gamma_{j}}$. Let $J=J_{1} \cup J_{2} \cup J_{3}$ where the sets are pairwise disjoint, and at least $J_{1}$ and $J_{3}$ are nonempty. Define the open Lipschitz surfaces $\Gamma(0), \Gamma, \Gamma(1) \subset \partial \Omega$ through their closures $\overline{\Gamma(0)}=\cup_{j \in J_{1}} \overline{\Gamma_{j}}, \bar{\Gamma}=\cup_{j \in J_{2}} \overline{\Gamma_{j}}$, and $\overline{\Gamma(1)}=\cup_{j \in J_{3}} \overline{\Gamma_{j}}$, respectively. Let $\alpha=\left\{\alpha_{j}\right\}_{j \in J_{2}} \subset(-\infty, 0]$ be a vector of dissipation parameters. 
Then the wave equation model (5.1) with equations

$$
\alpha_{j} \frac{\partial \phi}{\partial t}(\mathbf{r}, t)+\frac{\partial \phi}{\partial \nu}(\mathbf{r}, t)=0 \quad \text { for all } \mathbf{r} \in \Gamma_{j}, t \geq 0, \text { and } j \in J_{2}
$$

in place of the fourth equation in (5.1) defines the boundary node $\Xi_{\alpha}$ and the Hilbert spaces $\mathcal{X}, \mathcal{U}$, and $\mathcal{Z}_{\alpha}$ in a same way as presented in Section 5. Moreover, Theorem 5.1 and Corollary 5.2 (where $\alpha_{j}=0$ for all $j \in J_{2}$ ) hold without change.

In particular, the set $\Omega$ may be an union of a finite number of tubular domains described in Section 1. Even loops are possible and the interior domain dissipation can be added just like in Corollary 6.1. This configuration can be found in the study of the spectral limit behaviour of Neumann-Laplacian on graph-like structures in $[15,35]$.

Comments on the proof. The argument in Section 5 defines $\Xi_{\alpha}$, the Hilbert spaces $\mathcal{X}, \mathcal{U}$, and $\mathcal{Z}_{\alpha}$, and the GreenLagrange identity by splitting $\partial \Omega$ into three smooth components and patching things up using the results of Appendix A. The same can be done on any finite number of components since the results of Appendix A are sufficiently general to allow it. The solvability of the variational problems in the proof of Theorem 5.1 do not depend on the number of such boundary components either.

There is nothing in Section 5 that would exclude the further generalisation to $\Omega \subset \mathbb{R}^{n}$ for any $n \geq 2$ if standing assumptions (i)-(iv) in Appendix A remain true. If $n=2$ and $\Omega$ is a curvilinear polygon (i.e., it is simply connected), the necessary PDE toolkit can be found in Section 1 of [12].

Also Theorem 4.1 has extensions but not as many as Theorem 5.1. Firstly, the nonnegative constant $\alpha$ can be replaced by a nonnegative function $\alpha(\cdot) \in C[0,1]$ since the $s$-dependency is already present in the operator $D$ in (4.4). Secondly, strong boundary nodes described by Theorem 4.1 can be scaled to different interval lengths and coupled to finite transmission graphs as explained in [2] for impedance passive component systems. The full treatment of a simple transmission graph, consisting of three Webster's horn models in Y-configuration, has been given in Theorem 5.2 of [2]. More general finite configurations can be treated similarly, and the resulting impedance passive system can be translated to a scattering passive system by the external Cayley transform [30], Section 3, thus producing a generalisation of Theorem 4.1. We note that there is not much point in trying to derive the transmission graph directly from scattering passive systems since the continuity equation (for the pressure) and Kirchhoff's law (for the conservation of flow) at each node is easiest described by impedance notions.

That Theorem 3.2 cannot be used for all possible dissipation terms is seen by considering the wave equation with Kelvin-Voigt structural damping term

$$
\psi_{t t}=c^{2} \psi_{s s}+\frac{\partial}{\partial s}\left(\beta(s) \frac{\partial}{\partial s} \psi_{t}\right) \quad \text { where } \quad \beta(s) \geq 0 .
$$

For details of this dissipation model, see, e.g., [24]. To obtain the full dynamical system analogous to the one associated with Webster's equation, the same boundary and initial conditions can be used as in (1.3) for $\beta \in C^{\infty}[0,1]$ compactly supported $(0,1)$. Thus the operators $G_{W}$ and $K_{W}$ do not change. Following Section 4 we use the velocity potential and the pressure as state variables $\left[{ }_{\pi}^{\psi}\right]$. We define the Hilbert spaces $\mathcal{Z}_{W}$ and $\mathcal{X}_{W}$ similarly as well as the operators

$$
\begin{aligned}
L_{W} & :=\left[\begin{array}{cc}
0 & \rho^{-1} \\
\rho c^{2} \frac{\partial^{2}}{\partial s^{2}} & 0
\end{array}\right]: \mathcal{Z}_{W} \rightarrow \mathcal{X}_{W} \text { and } \\
\widetilde{H} & :=\left[\begin{array}{cc}
0 & 0 \\
0 \frac{\partial}{\partial s}\left(\beta(s) \frac{\partial}{\partial s}\right)
\end{array}\right]: \operatorname{dom}(\widetilde{H}) \subset \mathcal{X}_{W} \rightarrow \mathcal{X}_{W}
\end{aligned}
$$


where $\operatorname{dom}(\widetilde{H}):=H_{\{1\}}^{1}(0,1) \times\left\{f \in L^{2}(0,1): \beta(s) \frac{\partial f}{\partial s} \in H^{1}(0,1)\right\}$. The physical energy norm for $\mathcal{X}_{W}$ is given by (4.5) with $A(s)=\Sigma(s) \equiv 1$ representing a constant diameter straight tube. If the parameter $\beta \equiv 0$, the colligation $\left(G_{W}, L_{W}, K_{W}\right)$ is a special case of the conservative system $\Xi_{0}^{(W)}$ described in Theorem 4.1. Clearly, the domain of $\widetilde{H}$ cannot be further extended without violating the range inclusion in $\mathcal{X}_{W}$. On the other hand, the inclusion $\mathcal{Z} \subset \operatorname{dom}(\widetilde{H})$ required by Theorem 3.2 is not satisfied.

\section{Appendix A. Sobolev spaces and Green's identity}

We prove a sufficiently general form of Green's identity that holds in a tubular domain $\Omega$ (that has a Lipschitz boundary) with minimal assumptions on any functions involved. We make the following standing assumptions on $\Omega$ :

(i) $\Omega$ is a bounded Lipschitz domain so that $\Omega$ locally on one side of is boundary $\partial \Omega$;

(ii) there is a finite number of smooth, open, connected, and disjoint $(n-1)$-dimensional surfaces $\Gamma_{j}$ with the following property: the boundary $\partial \Omega$ is a union of all $\Gamma_{j}$ 's and parts of their common boundaries $\bar{\Gamma}_{j} \cap \bar{\Gamma}_{k}$ for $j \neq k$;

(iii) $\mathcal{H}^{n-2}\left(\bar{\Gamma}_{j} \cap \bar{\Gamma}_{k}\right)<\infty$ for all $j \neq k$ where $\mathcal{H}^{m}(M)$ is the $m$-dimensional Hausdorff measure for $1 \leq m \leq n$ of $M \subset \mathbb{R}^{n}$; and

(iv) for each $j$, there is a $C^{\infty}$ vector field $\nu_{j}$ defined in a neighbourhood of $\bar{\Omega}$ such that $\nu_{j}(\mathbf{r})$ is the exterior unit normal to $\Gamma_{j}$ at $\mathbf{r} \in \Gamma_{j}$.

That $\Gamma_{j} \subset \mathbb{R}^{n}$ is an open, bounded, and smooth $(n-1)$-dimensional surface means plainly the following: there is an open and bounded $\tilde{\Gamma}_{j} \subset \mathbb{R}^{n-1}$ and a $C^{\infty}$-diffeomorphism $\phi_{j}$ from $\tilde{\Gamma}_{j}$ onto $\Gamma_{j}$. The pair $\left(\phi_{j}, \tilde{\Gamma}_{j}\right)$ is a global coordinate representation of $\Gamma_{j}$.

The boundary conditions in Section 5 involve the Dirichlet conditions the end $\Gamma(1)$ of the tube, Neumann or Robin conditions on the wall $\Gamma$ of the tube, and a Robin condition on the end $\Gamma(0)$. In other words, we must impose different types of boundary conditions on the same connected component of $\partial \Omega$ which is in contrast with the restrictive technical assumptions on $\partial \Omega$ in, e.g., [17,29,43]. Such assumptions must be avoided in the verification of, e.g., the Green-Lagrange identity in Section 5 where we need a version of Green's identity suitable for wave propagation in a tubular domain with mixed boundary conditions. This is in Theorem A.3 below. The key fact ensuring the validity of this identity is that the interfaces where we switch between different boundary conditions are so small that Sobolev functions do not see them. That this is the case is a consequence of the Assumption (iii) above, and it is expressed rigorously in the following auxiliary result.

Lemma A.1. Let $\Omega$ be a bounded domain with a Lipschitz boundary, and let $E \subset \mathbb{R}^{n}$ be a compact set of zero capacity; i.e.,

$$
C(E):=\inf _{u \in S(E)} \int_{\mathbb{R}^{n}}\left(|u|^{2}+|\nabla u|^{2}\right) \mathrm{d} V=0
$$

where

$$
S(E):=\left\{u \in C^{\infty}\left(\mathbb{R}^{n}\right): 0 \leq u \leq 1 \text { in } \mathbb{R}^{n} \text { and } u=1 \text { in } N \text {, where } N \text { is open and } E \subset N\right\}
$$

Then

(i) the set $\mathcal{D}_{E}\left(\mathbb{R}^{n}\right)$ is dense in $H^{1}\left(\mathbb{R}^{n}\right)$ where

$$
\mathcal{D}_{E}\left(\mathbb{R}^{n}\right):=\left\{u \in \mathcal{D}\left(\mathbb{R}^{n}\right): u \text { vanishes in an open neighbourhood of } E\right\} ; \text { and }
$$

(ii) the set

is dense in $H^{1}(\Omega)$.

$$
\mathcal{D}_{E}(\bar{\Omega}):=\left\{\left.u\right|_{\Omega}: u \in \mathcal{D}_{E}\left(\mathbb{R}^{n}\right)\right\}
$$


Proof.

Claim (i): Let $u \in H^{1}\left(\mathbb{R}^{n}\right)$ and $\varepsilon>0$. Then by ([12], Thm. 1.4.2.1) there is $v \in \mathcal{D}\left(\mathbb{R}^{n}\right)$ such that $\|u-v\|_{H^{1}\left(\mathbb{R}^{n}\right)}<$ $\varepsilon / 2$.

By the vanishing capacity assumption (A.1), there is a sequence $\left\{\varphi_{j}\right\}_{j=1,2, \ldots} \subset C^{\infty}\left(\mathbb{R}^{n}\right)$ such that $\left.\varphi_{j}\right|_{N_{j}}=1$ for some neighbourhoods $N_{j}$ of $E$, and also

$$
\lim _{j \rightarrow \infty} \int_{\mathbb{R}^{n}}\left(\left|\varphi_{j}\right|^{2}+\left|\nabla \varphi_{j}\right|^{2}\right) \mathrm{d} V=0
$$

Defining $v_{j}(\mathbf{r}):=v(\mathbf{r})\left(1-\varphi_{j}(\mathbf{r})\right)$ we see that each of these functions satisfies $v_{j} \in \mathcal{D}_{E}\left(\mathbb{R}^{n}\right)$. It remains to prove that $\left\|v_{j}-v\right\|_{H^{1}\left(\mathbb{R}^{n}\right)}<\varepsilon / 2$ for all $j$ large enough, since then

$$
\left\|v_{j}-u\right\|_{H^{1}\left(\mathbb{R}^{n}\right)} \leq\left\|v_{j}-v\right\|_{H^{1}\left(\mathbb{R}^{n}\right)}+\|u-v\|_{H^{1}\left(\mathbb{R}^{n}\right)}<\varepsilon .
$$

By possibly replacing $\left\{\varphi_{j}\right\}_{j=1,2, \ldots}$ by its subsequence, we may assume that $\varphi_{j} \rightarrow 0$ pointwise almost everywhere (see [36], Thm. 3.12). Because $\left|v_{j}(\mathbf{r})\right| \leq|v(\mathbf{r})|$ for all $\mathbf{r} \in \mathbb{R}^{n}$ and $j=1,2, \ldots$, we have $v_{j} \rightarrow v$ in $L^{2}\left(\mathbb{R}^{n}\right)$ by the Lebesgue dominated convergence theorem. For the gradients, we note that $\nabla\left(v_{j}-v\right)=-\varphi_{j} \nabla v-v \nabla \varphi_{j}$. Thus $\left|\nabla\left(v_{j}-v\right)\right| \rightarrow 0$ in $L^{2}\left(\mathbb{R}^{n}\right)$, since both $\varphi_{j}$ and $\left|\nabla \varphi_{j}\right|$ tend to zero in $L^{2}\left(\mathbb{R}^{n}\right)$ by (A.3).

Claim (ii): Let $u \in H^{1}(\Omega)$ and take $\varepsilon>0$. Since $\Omega$ has a Lipschitz boundary, there is an extension operator $T \in \mathcal{L}\left(H^{1}(\Omega) ; H^{1}\left(\mathbb{R}^{n}\right)\right.$ ) such that $\left.(T u)\right|_{\Omega}=u$ (see [12], Thm. 1.4.3.1). By Claim (i), there is a function $v \in \mathcal{D}_{E}\left(\mathbb{R}^{n}\right)$ such that

$$
\left\|u-\left.v\right|_{\Omega}\right\|_{H^{1}(\Omega)} \leq\|T u-v\|_{H^{1}\left(\mathbb{R}^{n}\right)}<\varepsilon
$$

which completes the proof.

Let us review the Sobolev spaces and the boundary trace mappings on $\Omega$ and $\partial \Omega$ when the standing assumptions (i)-(iv) above hold. The boundary Sobolev spaces $H^{s}(\partial \Omega)$ and $H^{s}\left(\Gamma_{j}\right)$ for $s \in[-1,1]$ are defined as in ([12], Defs. 1.2.1.1 and 1.3.3.2). The zero extension Sobolev spaces on $\Gamma_{j}$ are defined by

$$
\tilde{H}^{s}\left(\Gamma_{j}\right):=\left\{u \in H^{s}\left(\Gamma_{j}\right): \tilde{u} \in H^{s}(\partial \Omega)\right\}
$$

for $s \in(0,1]$ where

$$
\tilde{u}(\mathbf{r}):= \begin{cases}u(\mathbf{r}) & \text { if } \mathbf{r} \in \Gamma_{j} \\ 0 & \text { if } \mathbf{r} \in \partial \Omega \backslash \Gamma_{j} .\end{cases}
$$

We use the Hilbert space norms $\|u\|_{\tilde{H}^{s}\left(\Gamma_{j}\right)}:=\|\tilde{u}\|_{H^{s}(\partial \Omega)}$. The space $\tilde{H}^{s}\left(\Gamma_{j}\right)$ is closed in this norm since restriction to $\Gamma_{j}$ from $\partial \Omega$ is a bounded operator from $H^{s}(\partial \Omega)$ to $H^{s}\left(\Gamma_{j}\right)$ for $0 \leq s \leq 1$. This boundedness follows trivially by restriction using the Gagliardo norm (see [12], Eq. (1,3,3,3), p. 20). Then $H^{s}(\partial \Omega) \subset L^{2}(\partial \Omega)$ and $\tilde{H}^{s}\left(\Gamma_{j}\right) \subset H^{s}\left(\Gamma_{j}\right) \subset L^{2}\left(\Gamma_{j}\right)$ with bounded inclusions.

The Dirichlet trace operator $\gamma$ is first defined for functions $f \in \mathcal{D}(\bar{\Omega})$ simply by restriction $\gamma f:=\left.f\right|_{\partial \Omega}$. This operator has a unique extension to a bounded operator $\gamma \in \mathcal{L}\left(H^{1}(\Omega) ; H^{1 / 2}(\partial \Omega)\right)$; see ([12], Thm. 1.5.1.3) and Lemma A.1. All this holds for any Lipschitz domain $\Omega$.

We define the Neumann trace operator separately on each surface $\Gamma_{j}$ using the vector fields $\nu_{j}$. Such an operator $\gamma_{j} \frac{\partial}{\partial \nu_{j}}$ is first defined on $\mathcal{D}(\bar{\Omega})$ (with values in $L^{2}(\partial \Omega)$ ) by setting $\left(\gamma_{j} \frac{\partial}{\partial \nu_{j}} f\right)(\mathbf{r}):=\nu_{j}(\mathbf{r}) \cdot \nabla f(\mathbf{r})$ for all $\mathbf{r} \in \Gamma_{j}$; here $\gamma_{j} f:=\left.f\right|_{\Gamma_{j}}$ and $\frac{\partial}{\partial \nu_{j}}:=\nu_{j} \cdot \nabla$. It is easy to see that $\frac{\partial f}{\partial \nu_{j}} \in H^{1}(\Omega)$ and hence $\gamma_{j} \frac{\partial}{\partial \nu_{j}}$ has an extension to an operator in $\mathcal{L}\left(H^{2}(\Omega) ; H^{1 / 2}\left(\Gamma_{j}\right)\right)$ by ([12], Thm. 1.5.1.3). We then define the full Neumann trace operator $\gamma \frac{\partial}{\partial \nu}$ on $\cup_{j} \Gamma_{j}$ by

$$
\gamma \frac{\partial f}{\partial \nu}(\mathbf{r}):=\gamma_{j} \frac{\partial f}{\partial \nu_{j}}(\mathbf{r}) \quad \text { for all } f \in H^{2}(\Omega) \quad \text { and (almost) all } \quad \mathbf{r} \in \Gamma_{j}
$$


Note that the function $\gamma \frac{\partial f}{\partial \nu}$ is not defined at all on the exceptional set of capacity zero

$$
E:=\cup_{j \neq k}\left(\bar{\Gamma}_{j} \cap \bar{\Gamma}_{k}\right)
$$

of the non-smooth part of $\partial \Omega$. That $C(E)=0$ follows from the standing assumption (iii) by ([6], Thm. 3, p. 154).

We need to extend each $\gamma_{j} \frac{\partial}{\partial \nu_{j}}$ to the Hilbert space

$$
E\left(\Delta ; L^{2}(\Omega)\right):=\left\{f \in H^{1}(\Omega): \Delta f \in L^{2}(\Omega)\right\}
$$

that is equipped with the norm defined by $\|f\|_{E\left(\Delta ; L^{2}(\Omega)\right)}^{2}=\|f\|_{H^{1}(\Omega)}^{2}+\|\Delta f\|_{L^{2}(\Omega)}^{2}$.

Proposition A.2. Let the domain $\Omega \subset \mathbb{R}^{n}$ satisfy the standing assumptions (i)-(iv).

(i) Then each Neumann trace operator $\gamma_{j} \frac{\partial}{\partial \nu_{j}}$ (originally defined on $\mathcal{D}(\bar{\Omega})$ ) has a unique extension (also denoted by $\left.\gamma_{j} \frac{\partial}{\partial \nu_{j}}\right)$ that is bounded from $E\left(\Delta ; L^{2}(\Omega)\right)$ into the dual space of $\tilde{H}^{1 / 2}\left(\Gamma_{j}\right)$ with pivot space $L^{2}\left(\Gamma_{j}\right)$.

(ii) We have

$$
\int_{\Omega}(\Delta u) v \mathrm{~d} V+\int_{\Omega} \nabla u \cdot \nabla v \mathrm{~d} V=\sum_{j}\left\langle\gamma_{j} \frac{\partial u}{\partial \nu}, \gamma_{j} v\right\rangle_{\left[\tilde{H}^{1 / 2}\left(\Gamma_{j}\right)\right]^{d}, \tilde{H}^{1 / 2}\left(\Gamma_{j}\right)}
$$

for all $u \in E\left(\Delta ; L^{2}(\Omega)\right)$ and $v \in H^{1}(\Omega)$ such that $\gamma_{j} v \in \tilde{H}^{1 / 2}\left(\Gamma_{j}\right)$ for all $j$.

Proof. The classical Green's identity for $u \in \mathcal{D}(\bar{\Omega})$ and $v \in \mathcal{D}_{E}(\bar{\Omega})$ is

$$
\int_{\Omega}(\Delta u) v \mathrm{~d} V+\int_{\Omega} \nabla u \cdot \nabla v \mathrm{~d} V=\sum_{j} \int_{\Gamma_{j}} \gamma_{j} \frac{\partial u}{\partial \nu_{j}} \gamma_{j} v \mathrm{~d} A
$$

where $E$ is the exceptional set in (A.5). Indeed, since $v$ vanishes near the interfaces $\bar{\Gamma}_{j} \cap \bar{\Gamma}_{k}$ for $j \neq k$, we may initially apply Green's identity just like (A.6) but over a subdomain of $\Omega$ that has been obtained from $\Omega$ by rounding slightly at all $\partial \Gamma_{j}$ 's but preserving essentially all of $\partial \Omega$. Here the functions $u$ and $v$ and the rounded subdomain are all smooth, so the Green's formula we use here is the one familiar from vector calculus. Then we get (A.6) by rewriting the result as integrals over the original $\Omega$ and the original boundary pieces $\Gamma_{j}$, noting that on additional points the integrands vanish because $v \in \mathcal{D}_{E}(\bar{\Omega})$. Notice that all the functions appearing above are smooth, so we can use Green's formula

It follows from (A.6) that we have for $u \in \mathcal{D}(\bar{\Omega})$ and $v \in \mathcal{D}_{E}(\bar{\Omega})$ the estimate

$$
\left|\sum_{j}\left\langle\gamma_{j} \frac{\partial u}{\partial \nu_{j}}, \gamma_{j} v\right\rangle_{L^{2}\left(\Gamma_{j}\right)}\right| \leq\|u\|_{E\left(\Delta ; L^{2}(\Omega)\right)} \cdot 4\|v\|_{H^{1}(\Omega)} .
$$

Because $\mathcal{D}_{E}(\bar{\Omega})$ is dense in $H^{1}(\Omega)$ by Lemma A.1 and $\gamma \in \mathcal{L}\left(H^{1}(\Omega) ; H^{1 / 2}(\partial \Omega)\right)$ by the trace Theorem $([12]$, Thm. 1.5.1.3), we conclude that (A.7) holds for all $u \in \mathcal{D}(\bar{\Omega})$ and $v \in H^{1}(\Omega)$.

Fix now $j$ and $g \in \tilde{H}^{1 / 2}\left(\Gamma_{j}\right)$, and define $\tilde{g} \in H^{1 / 2}(\partial \Omega)$ by (A.4). Because the Dirichlet trace $\gamma: H^{1}(\Omega) \rightarrow$ $H^{1 / 2}(\partial \Omega)$ is bounded and surjective, it has a continuous right inverse $P \in \mathcal{L}\left(H^{1 / 2}(\partial \Omega) ; H^{1}(\Omega)\right.$ ) (see [12], Thm. 1.5.1.3). Thus we have the estimate $4\|P g\|_{H^{1}(\Omega)} \leq K\|\tilde{g}\|_{H^{1 / 2}(\partial \Omega)}=K\|g\|_{\tilde{H}^{1 / 2}\left(\Gamma_{j}\right)}$ for all $g \in \tilde{H}^{1 / 2}\left(\Gamma_{j}\right)$.

It follows from all this and (A.7) that we have

$$
\left|\Phi_{g}(u)\right| \leq K\|u\|_{E\left(\Delta ; L^{2}(\Omega)\right)} \cdot\|g\|_{\tilde{H}^{1 / 2}\left(\Gamma_{j}\right)}
$$

for all $g \in \tilde{H}^{1 / 2}\left(\Gamma_{j}\right)$ where $\Phi_{g}(u):=\left\langle\gamma \frac{\partial u}{\partial \nu}, \tilde{g}\right\rangle_{L^{2}(\partial \Omega)}=\left\langle\gamma_{j} \frac{\partial u}{\partial \nu_{j}}, g\right\rangle_{L^{2}\left(\Gamma_{j}\right)}$ for $u \in \mathcal{D}(\bar{\Omega})$. Since $\mathcal{D}(\bar{\Omega})$ is dense in $E\left(\Delta ; L^{2}(\Omega)\right)$ by ([12], Lem. 1.5.3.9), we may extend $\Phi_{g}, g \in \tilde{H}^{1 / 2}\left(\Gamma_{j}\right)$, by continuity to a continuous linear functional on $E\left(\Delta ; L^{2}(\Omega)\right)$ satisfying estimate (A.8), too. 
For each fixed $u \in E\left(\Delta ; L^{2}(\Omega)\right)$, the mapping $g \mapsto \Phi_{g}(u)$ is a continuous linear functional on $\tilde{H}^{1 / 2}\left(\Gamma_{j}\right)$ by (A.8). Hence, there is a representing vector - denoted by $\gamma_{j} \frac{\partial u}{\partial \nu_{j}}-$ in the dual space $\left[\tilde{H}^{1 / 2}\left(\Gamma_{j}\right)\right]^{d}$ such that $\Phi_{g}(u)=\left\langle\gamma_{j} \frac{\partial u}{\partial \nu_{j}}, g\right\rangle_{\left[\tilde{H}^{1 / 2}\left(\Gamma_{j}\right)\right]^{d}, \tilde{H}^{1 / 2}\left(\Gamma_{j}\right)}$. This proves Claim (i). Claim (ii) follows by a density argument using Claim (i) and (A.8).

Theorem A.3 (Green's identity). Let the domain $\Omega \subset \mathbb{R}^{n}$ satisfy the standing assumptions (i)-(iv) above. Assume that $u \in H^{1}(\Omega)$ is such that $\Delta u \in L^{2}(\Omega)$ and satisfies $\gamma \frac{\partial u}{\partial \nu} \in L^{2}\left(\cup_{j=1}^{k} \Gamma_{j}\right)$ for some $1 \leq k \leq n$. Then the Green's identity

$$
\int_{\Omega}(\Delta u) v \mathrm{~d} V+\int_{\Omega} \nabla u \cdot \nabla v \mathrm{~d} V=\sum_{j=1}^{k} \int_{\Gamma_{j}} \frac{\partial u}{\partial \nu} v \mathrm{~d} A+\sum_{j=k+1}^{n}\left\langle\gamma_{j} \frac{\partial u}{\partial \nu_{j}}, \gamma_{j} v\right\rangle_{\left[\tilde{H}^{1 / 2}\left(\Gamma_{j}\right)\right]^{d}, \tilde{H}^{1 / 2}\left(\Gamma_{j}\right)}
$$

holds for functions $v \in H^{1}(\Omega)$ such that $\gamma_{j} v \in \tilde{H}^{1 / 2}\left(\Gamma_{j}\right)$ for $k+1 \leq j \leq n$.

For $n=2$, this is a generalisation of ([12], Thm. 1.5.3.11). See also [12], discussion on page 62 for domains with $C^{1,1}$-boundaries. The assumption $\gamma \frac{\partial u}{\partial \nu} \in L^{2}\left(\cup_{j=1}^{k} \Gamma_{j}\right)$ simply means that $\gamma_{j} \frac{\partial u}{\partial \nu_{j}} \in L^{2}\left(\Gamma_{j}\right)$ for all $j=1,2, \ldots, k$ where $\gamma_{j} \frac{\partial u}{\partial \nu_{j}}$ is understood as an element of $\left[\tilde{H}^{1 / 2}\left(\Gamma_{j}\right)\right]^{d}$ which includes $L^{2}\left(\Gamma_{j}\right)$; see Proposition A.2.

Proof. As explained above, we have $\gamma_{j} v, \gamma_{j} \frac{\partial u}{\partial \nu_{j}} \in L^{2}\left(\Gamma_{j}\right)$ for all $j=1, \ldots, k$. Then (A.9) follows from Claim (ii) of Proposition A.2 under the additional assumption that $\gamma_{j} v \in \tilde{H}^{1 / 2}\left(\Gamma_{j}\right)$ for all $j$. The functions in $\mathcal{D}_{E}(\bar{\Omega})$ clearly satisfy this additional assumption, and they are dense in $H^{1}(\Omega)$. This proves the claim.

An alternative to the above piecewise construction is to start with the global Neumann trace $\gamma \frac{\partial}{\partial \nu} u$ defined for $u \in E\left(\Delta ; L^{2}(\Omega)\right)$ with values in $H^{-1 / 2}(\partial \Omega)$ (see, e.g. [45], Thm. 13.7.6). The global Neumann trace $\gamma \frac{\partial}{\partial \nu} u$ can be restricted to the spaces $\tilde{H}^{1 / 2}\left(\Gamma_{j}\right)$, and Claim (ii) of Proposition A.2 follows from a global Green's identity in a general Lipschitz domain. However, one still needs Lemma A.1 to prove Theorem A.3.

It remains to prove the Poincaré inequality that is used to show that the expression (5.7) is a valid Hilbert space norm for the state space. Let $\Gamma_{j}$ be one of the boundary components of $\partial \Omega$ as described above. By the standing assumptions (i), (ii) given in the beginning of this appendix, the set $\Gamma_{j}$ has a finite, positive area $A_{j}=\int_{\Gamma_{j}} \mathrm{~d} A$. Thus, we can define the mean value operator $M_{j}: H^{1}(\Omega) \rightarrow \mathbb{C}$ on $\Gamma_{j}$ by

$$
M_{j} u=\frac{1}{A_{j}} \int_{\Gamma_{j}} \gamma_{j} u \mathrm{~d} A,
$$

It is clear that $M_{j}$ is a bounded linear functional on $H^{1}(\Omega)$, and we may regard it as an element of $\mathcal{L}\left(H^{1}(\Omega)\right)$ satisfying $M_{j}^{2}=M_{j}$ by considering $M_{j} u$ as a constant function on $\Omega$.

Theorem A.4 (Poincaré inequality). Let the domain $\Omega \subset \mathbb{R}^{n}$ satisfy the standing assumptions (i)-(iv) above, and let $\Gamma_{j}$ be one of the boundary components of $\partial \Omega$. There is a constant $C<\infty$ such that

$$
\left\|u-M_{j} u\right\|_{L^{2}(\Omega)} \leq C\|\nabla u\|_{L^{2}(\Omega)}
$$

for all $u \in H^{1}(\Omega)$. Thus, we have $\|u\|_{L^{2}(\Omega)} \leq C\|\nabla u\|_{L^{2}(\Omega)}$ for $u \in H^{1}(\Omega) \cap \operatorname{ker}\left(\gamma_{j}\right)$.

Proof. The argument is a standard argument by contradiction using the Rellich-Kondrachov compactness theorem (see e.g. [6], Thm. 1, p. 144). For a contradiction against (A.10), assume that there exist functions $u_{k} \in H^{1}(\Omega)$ such that there is the strict inequality

$$
\left\|u_{k}-M_{j} u_{k}\right\|_{L^{2}(\Omega)}>k\left\|\nabla u_{k}\right\|_{L^{2}(\Omega)} \quad \text { for } \quad k=1,2, \ldots
$$


None of the functions $u_{k}$ are constant functions since for such functions (A.10) holds for any $C \geq 0$. So, we can define the functions

$$
v_{k}:=\frac{u_{k}-M_{j} u_{k}}{\left\|u_{k}-M_{j} u_{k}\right\|_{L^{2}(\Omega)}}
$$

satisfying for all $k$ the normalisation $\left\|v_{k}\right\|_{L^{2}(\Omega)}=1$ and also $M_{j} v_{k}=0$ by using $M_{j}^{2}=M_{j}$. Since

$$
\left\|\nabla v_{k}\right\|^{2}=\frac{\left\|\nabla u_{k}\right\|_{L^{2}(\Omega)}^{2}}{\left\|u_{k}-M_{j} u_{k}\right\|_{L^{2}(\Omega)}^{2}}<\frac{1}{k^{2}}
$$

by the counter assumption, we get

$$
\left\|v_{k}\right\|_{H^{1}(\Omega)}^{2}=\left\|v_{k}\right\|_{L^{2}(\Omega)}^{2}+\left\|\nabla v_{k}\right\|_{L^{2}(\Omega)}^{2} \leq 1+\frac{1}{k^{2}} \leq 2 .
$$

Since the embedding $H^{1}(\Omega) \subset L^{2}(\Omega)$ is compact (by the boundedness of $\Omega$ and the Rellich-Kondrachov compactness theorem, see e.g. [6], Thm. 1, p. 144), we have a function $v$ such that $v_{k} \rightarrow v$ in $L^{2}(\Omega)$ by possibly replacing $\left\{v_{k}\right\}$ by its subsequence. Moreover, $\|v\|_{L^{2}(\Omega)}=1$ since $\left\|v_{k}\right\|_{L^{2}(\Omega)}=1$ for all $k$.

Since $\left\|\nabla v_{k}\right\|_{L^{2}(\Omega)} \leq 1 / k$, we see that $v_{k} \rightarrow v$ in $H^{1}(\Omega)$ and hence $\nabla v=0$. Thus $v$ is a constant function. Because $M_{j} v=\lim _{k \rightarrow \infty} M_{j} v_{k}=0$, we conclude that $v=0$ which contradicts the fact that $\|v\|_{L^{2}(\Omega)}=1$. This proves (A.10), and the Poincaré equality follows trivially from this.

Acknowledgements. The authors have received support from the Finnish Graduate School on Engineering Mechanics, the Norwegian Research Council, and Aalto Starting Grant (grant no. 915587). The authors wish to thank the anonymous referees for many valuable comments.

\section{REFERENCES}

[1] A. Aalto, D. Aalto, J. Malinen and M. Vainio, Modal locking between vocal fold and vocal tract oscillations. Preprint arXiv: 1211.4788 (2012).

[2] A. Aalto and J. Malinen, Composition of passive boundary control systems. Math. Control Relat. Fields 3 (2013) 1-19.

[3] D. Aalto, O. Aaltonen, R.-P. Happonen, P. Jääsaari, A. Kivelä, J. Kuortti, J.M. Luukinen, J. Malinen, T. Murtola, R. Parkkola, J. Saunavaara and M. Vainio, Large scale data acquisition of simultaneous MRI and speech. Appl. Acoustics 83 (2014) 64-75.

[4] J. Cervera, A.J. van der Schaft and A. Baños, Interconnection of port-Hamiltonian systems and composition of Dirac structures. Automatica 43 (2007) 212-225.

[5] E. Eisner, Complete solutions of the "Webster" horn equation. J. Acoust. Soc. Am. 41 (1967) 1126-1146.

[6] L. Evans and R. Gariepy, Measure Theory and the Fine Properties of Functions. CRC Press (1992).

[7] H. Fattorini, Boundary control systems. SIAM J. Control 6 (1968) 349-385.

[8] A. Fetter and J. Walecka, Theoretical Mechanics of Particles and Continua. Dover (2003).

[9] F. Flandoli, I. Lasiecka and R. Triggiani, Algebraic Riccati equations with non-smoothing observation arising in hyperbolic and Euler-Bernoulli boundary control problems. Annal. Math. Pura Appl. CLIII (1988) 307-382.

[10] F. Gesztesy and H. Holden, The damped string problem revisited. J. Differ. Equ. 251 (2011) 1086-1127.

[11] V. Gorbachuk and M. Gorbachuk, Boundary Value Problems for Operator Differential Equations, vol. 48 of Math. Appl. (Soviet Ser.). Kluwer Academic Publishers Group, Dordrecht (1991).

[12] P. Grisvard, Elliptic Problems in Non-Smooth Domains. Pitman (1985).

[13] A. Hannukainen, T. Lukkari, J. Malinen and P. Palo, Vowel formants from the wave equation. J. Acoust. Soc. Am. Express Lett. 122 (2007) EL1-EL7.

[14] T. Kato, Perturbation Theory for Linear Operators, vol. 132 of Grundlehren der Mathematischen Wissenschaften. Springer Verlag (1980).

[15] P. Kuchment and H. Zeng, Convergence of spectra of mesoscopic systems collapsing onto a graph. J. Math. Anal. Appl. 258 (2001) 671-700.

[16] M. Kurula, H. Zwart, A. J. van der Schaft and J. Behrndt, Dirac structures and their composition on Hilbert spaces. J. Math. Anal. Appl. 372 (2010) 402-422.

[17] J. Lagnese, Decay of solutions of wave equations in a bounded region with boundary dissipation. J. Differ. Equ. 50 (1983) 163-182.

[18] I. Lasiecka, J.L. Lions and R. Triggiani, Nonhomogenous boundary value problems for second order hyperbolic operations. J. Math. Pures Appl. 65 (1986) 149-192. 
[19] I. Lasiecka and R. Triggiani, Control Theory for Partial Differential Equations: Continuous and Approximation Theories. II. Abstract hyperbolic-like systems, over a finite time horizon, vol. 75 of Encycl. Math. Appl. Cambridge University Press, Cambridge (2000).

[20] M. Lesser and J. Lewis, Applications of matched asymptotic expansion methods to acoustics. I. The Webster horn equation and the stepped duct. J. Acoust. Soc. Am. 51 (1971) 1664-1669.

[21] M. Lesser and J. Lewis, Applications of matched asymptotic expansion methods to acoustics. II. The open-ended duct. J. Acoust. Soc. Am. 52 (1972) 1406-1410.

[22] J. L. Lions, Exact controllability, stabilization and perturbations for distributed systems. SIAM Rev. 30 (1988) 1-68.

[23] J. L. Lions and E. Magenes, Non-Homogenous Boundary Value Problems and Applications II, vol. 182 of Die Grundlehren der Mathematischen Wissenchaften. Springer Verlag, Berlin (1972).

[24] K. Liu and Z. Liu, Exponential decay of energy of vibrating strings with local viscoelasticity. Z. Angew. Math. Phys. 53 (2002) 265-280.

[25] T. Lukkari and J. Malinen, A posteriori error estimates for Webster's equation in wave propagation (2011), manuscript.

[26] T. Lukkari and J. Malinen, Webster's equation with curvature and dissipation (2011). Preprint arXiv:1204.4075.

[27] J. Malinen, Conservativity of time-flow invertible and boundary control systems, Technical Report A479. Helsinki University of Technology Institute of Mathematics (2004).

[28] J. Malinen, O. Staffans and G. Weiss, When is a linear system conservative? Quart. Appl. Math. 64 (2006) 61-91.

[29] J. Malinen and O. Staffans, Conservative boundary control systems. J. Differ. Equ. 231 (2006) $290-312$.

[30] J. Malinen and O. Staffans, Impedance passive and conservative boundary control systems. Complex Anal. Oper. Theory 2 (2007) 279-300.

[31] A. Nayfeh and D. Telionis, Acoustic propagation in ducts with varying cross sections. J. Acoust. Soc. Am. 54 (1973) $1654-1661$.

[32] S. Rienstra, Sound transmission in slowly varying circular and annular lined ducts with flow. J. Fluid Mech. 380 (1999) 279-296.

[33] S. Rienstra, Webster's horn equation revisited. SIAM J. Appl. Math. 65 (2005) 1981-2004.

[34] S. Rienstra and W. Eversman, A numerical comparison between the multiple-scales and finite-element solution for sound propagation in lined flow ducts. J. Fluid Mech. 437 (2001) 367-384.

[35] J. Rubinstein and M. Schatzman, Variational problems on multiply connected thin strips I: Basic estimates and convergence of the Laplacian spectrum. Arch. Ration. Mech. Anal. 160 (2001) 271-308.

[36] W. Rudin, Real and Complex Analysis. McGraw-Hill Book Company, New York, 3rd edition (1986).

[37] D. Russell, Controllability and stabilizability theory for linear partial differential equations: recent progress and open questions. SIAM Rev. 20.

[38] D. Salamon, Infinite dimensional linear systems with unbounded control and observation: a functional analytic approach. Trans. Amer. Math. Soc. 300 (1987) 383-431.

[39] D. Salamon, Realization theory in Hilbert spaces. Math. Systems Theory 21 (1989) 147-164.

[40] V. Salmon, Generalized plane wave horn theory. J. Acoust. Soc. Am. 17 (1946) 199-211.

[41] V. Salmon, A new family of horns. J. Acoust. Soc. Am 17 (1946) 212-218.

[42] O. Staffans, Well-Posed Linear Systems. Cambridge University Press, Cambridge (2004).

[43] R. Triggiani, Wave equation on a bounded domain with boundary dissipation: An operator approach. J. Math. Anal. Appl. 137 (1989) 438-461.

[44] M. Tucsnak and G. Weiss, How to get a conservative well-posed linear system out of thin air. II. Controllability and stability. SIAM J. Control Optim. 42 (2003) 907-935.

[45] M. Tucsnak and G. Weiss, Observation and Control for Operator Semigroups. Birkhäuser Verlag, Basel (2009).

[46] J. Villegas, A Port-Hamiltonian Approach to Distributed Parameter Systems, Ph.D. thesis, University of Twente (2007).

[47] A. Webster, Acoustic impedance, and the theory of horns and of the phonograph. Proc. Natl. Acad. Sci. USA 5 (1919) $275-282$.

[48] G. Weiss and M. Tucsnak, How to get a conservative well-posed linear system out of thin air. I. Well-posedness and energy balance. ESAIM: COCV 9 (2003) 247-274. 